University of Konstanz

Department of Economics

\title{
Regulation in the Market for Education and Optimal Choice of Curriculum
}

Gerald Eisenkopf and Ansgar Wohlschlegel

Working Paper Series

2011-16 


\title{
Regulation in the Market for Education and Optimal Choice of Curriculum ${ }^{\text {th }}$
}

\author{
Gerald Eisenkopf ${ }^{\mathrm{a}}$, Ansgar Wohlschlegel ${ }^{\mathrm{b}}$ \\ ${ }^{a}$ Department of Economics, University of Konstanz, Box 131, 78457 Konstanz, Germany \\ Thurgau Institute of Economics, Hauptstr. 90, 8280 Kreuzlingen, Switzerland. \\ eisenkopf@twi-kreuzlingen.ch. \\ ${ }^{b}$ Wirtschaftspolitische Abteilung, University of Bonn, Adenauerallee 24-42, 53113 Bonn, Germany. \\ wohlschlegel@uni-bonn.de.
}

\begin{abstract}
We analyze educational institutions' incentives to set up demanding or lax curricula in duopolistic markets for education with endogenous enrolment of students. We assume that there is a positive externality of student achievement on the local economy. Comparing the case of regulated tuition fees with an unregulated market, we identify the following inefficiencies: Under regulated tuition fees schools will set up inefficiently lax curricula in an attempt to please low-quality students even if schools internalize some of the externality. On the other hand, unregulated schools set up excessively differentiated curricula in order to relax competition in tuition fees. Deregulation gets more attractive if a larger fraction of the externality is internalized.
\end{abstract}

Keywords: Education, Local Externalities, Product Differentiation, Price Competition, Vouchers

\section{Introduction}

Schools and universities provide significant inputs to local and regional economies. However, some of these benefits of education are externalities: Aggregate student achievement spills over into the productivity of the entire local economy through various channels such as human capital spillovers (Rauch (1993), Rosenthal and Strange (2008), Andersson et al. (2009)), facilitating education of others (Benabou (1993)) or reducing crime (Machin et al. (2011)). Hence, student achievement produced by educational institutions is a local public good.

At the same time, student achievement produced by an educational institution has an impact on its competitive position in the market for education. These strategic considerations are unlikely to be perfectly aligned with social benefits from student achievement. Hence,

\footnotetext{
«The authors would like to thank an anonymous referee, the editor, Lisa Bruttel, Eberhard Feess, Philipp Harms and seminar participants in Aachen, Cologne and at the Economics of Education Committee of the Verein für Socialpolitk for helpful comments.
} 
a great part of the public is skeptical towards an increasing importance of market forces in education. People are, for instance, concerned that unregulated profit maximization of universities might lead to under-provision or inefficient allocation of quality and quantity due to imperfect competition, externalities or imperfections in the market for funds. ${ }^{1} \mathrm{~A}$ different concern repeatedly raised in the public discussion is that a market based university system may induce universities to be excessively slack on study requirements due to competition for paying students. ${ }^{2}$ This paper's aim is to provide a first study on educational institutions' incentives to set up demanding or lax curricula under two different market regimes: an unregulated market in which educational institutions compete in tuition fees and curricula, and a market in which tuition fees are regulated to a fixed level, so that competition takes place only in curricula.

Our analysis is driven by an assumption which we believe is the distinctive feature of the choice of curriculum as opposed to, for instance, the choice of teaching quality: that a more demanding curriculum is more productivity enhancing but more costly to students than a less demanding one and, assuming that students have different abilities, a higher ability reduces a student's marginal cost of preparing the exam of a more demanding curriculum. Hence, low-ability students will prefer lax over demanding curricula even if tuition fees are identical.

When dealing with the aforementioned externality of student achievement, we will allow for the possibility that educational institutions directly take it into account to some extent: Curricula of primary and secondary schools are in many countries at least influenced by local school boards and will thus internalize local externalities such as reduced crime rates. On the other hand, even private universities may care somewhat about the human capital spillover to the local population, as they recruit much of their student body from the local economy. ${ }^{3}$ We will label the total externality of student achievement social teaching spillover and the part of it that is internalized by schools private teaching spillover.

Contradicting the conjectures made by some media, we find that in an unregulated market some schools and universities will set up even inefficiently tough curricula in order to relax subsequent competition in tuition fees. In contrast, it is regulated tuition fees which tend to induce all institutions to be excessively lax on students. Intuitively, the equilibrium curriculum in the latter regime must necessarily be located 'at the median student' among all enrolled students, as otherwise unilateral slight underbidding or overbidding would give the deviating institution more than half of total demand and thus increase its profit, whereas this slight deviation leaves the deviating institution's private teaching spillover roughly constant. Hence, in equilibrium even the private teaching spillover is not taken into account at all. In other words, the teaching spillover works like a positive externality, so that the equilibrium curriculum will be too lax, and this would be true even if all of the spillover was taken into

\footnotetext{
${ }^{1}$ Some of these issues have been taken up by theoretical economic research, e.g. Epple and Romano (1998, 2008), Hoxby (1999), Del Rey (2001), Kemnitz (2007).

${ }^{2}$ The Economist (1998, Sep 18th).

${ }^{3}$ See, for instance, Jepsen and Montgomery (2009)
} 
account in schools' objective functions. On the other hand, equilibrium curricula do depend on the private benefit. Hence, the unregulated market will be more attractive the larger the fraction of the private teaching spillover in social spillover.

Most assumptions we make in our model are standard in the literature on product differentiation. Technically speaking, preferences in our model combine aspects of vertical and horizontal differentiation, the difference between which is that consumers of a vertically differentiated good agree that higher quality is better but disagree with respect to their willingness to pay for quality, ${ }^{4}$ whereas in the case of horizontal differentiation consumers bear preference costs whenever the good differs from their preferred variety in any direction. ${ }^{5}$ In particular, our result of excessively tough curricula in the unregulated case corresponds to standard results in the literature on vertical differentiation. On the other hand, the possibility of profitably underbidding a rival's curriculum, which is a main driving force of the inefficiency associated with regulated tuition fees, crucially depends on the horizontal-differentiation aspect of our model.

To the best of our knowledge, we are the first to analyze incentives for educational institutions to set up demanding or lax curricula. There is a line of literature on educational institutions competing in quality and tuition fees. ${ }^{6}$ The main differences between the choices of quality and curricula are that (i) for arguments based on quality a model of purely vertical differentiation would be appropriate, and (ii) the practical discussion on quality is always closely related to cost arguments, whereas in our argument making exams harder to pass does not necessarily involve higher costs for schools. Epple and Romano (1998) and Epple et al. (2002) analyze competition between schools with different objectives, public and private schools, and find that private schools skim off the wealthiest and most able students, an effect which is more pronounced in a system of universal vouchers. By contrast, Epple and Romano (2008) show that this negative side effect of competition can be mitigated by appropriate design of the voucher system. While these papers focus mainly on distributional considerations, Hoxby (1999) shows that competition among school districts increases the efficiency of the use of public funds in education.

The models most closely related to ours are Del Rey (2001) and Kemnitz (2007), who also analyze the market for education within a framework of spatial competition. Del Rey (2001) analyzes universities' choices to devote funds to teaching or research in a model which is institutionally similar to our regulated case: There is non-price competition between universities, and the budget allocated to universities by the government depends (in part) on the number of students. Unlike in our model, however, universities are horizontally differentiated in a dimension which is unrelated to education. Quality then takes on the role of the price in the standard Hotelling model. We take up a different dimension of an educational institution's strategy space, the choice of curriculum, and seek to assess welfare

\footnotetext{
${ }^{4}$ See for instance Shaked and Sutton (1982).

${ }^{5}$ An example is the model by Hotelling (1929).

${ }^{6}$ However, quality improvements which are effective in enhancing student achievement may be difficult to find in practice. For instance, Buddin and Zamarro (2009) show that there are large differences in student achievement across teachers, but they cannot be pinned down to traditional measures of teacher quality.
} 
implications of our results, especially in comparison to the case of price competition. In this respect, our work can be seen as complementary to Del Rey (2001).

Kemnitz (2007) analyzes universities' quality incentives within a standard model of vertical differentiation. Under the assumption of a fixed total number of students in the economy, he shows that the optimal uniform-fee regulation always outperforms the unregulated market outcome. The main reason for this result is that students agree that higher quality is always better, so that under regulated tuition fees universities will outbid each other in quality until the marginal cost of doing so is equal to the tuition fee (or until the maximum quality is reached). Consequently, the equilibrium in that model is the efficient outcome among all symmetric outcomes. This result is dramatically different to our Proposition 3: In our model there are always some students who would prefer a less demanding curriculum. Hence, a school/university offering a tough curriculum exposes itself to the risk of being slightly undercut and end up with less than half of total demand. This difference is the main reason why our conclusions are over all much less in favor of regulated tuition fees than in the preceding literature.

Other theoretical work on optimal policies for universities to attract students has mainly focused on peer-group effects inducing universities to go after especially the high-ability students. In Rothschild and White (1995) and Caucutt (2002), this leads to price discrimination, whereas De Fraja and Iossa (2002) analyze competition through admission standards. Gary-Bobo and Trannoy (2008) then explain why a monopolistic university would combine admission exams with tuition fees.

Our comparison of unregulated price competition with regulated tuition fees is motivated by the fact that both systems exist in reality. While price competition both in secondary and higher education is regulated or eliminated in most European countries, the US are probably the chief example for price competition among universities. Empirical papers on US data give strong support for most features of the existing models on competition in tuition fees, as for instance Epple, Romano, and Sieg (2003, 2006). Competition in secondary education takes more subtle forms on both sides of the Atlantic but does exist and is largely beneficial according to several empirical studies such as Hoxby (2000) and Rincke (2006) for the US, Gibbons et al. (2008) for England and Wößmann (2003) for Europe. Such competition in secondary education is usually not in price but rather in quality and works through a voucher system. In a voucher system, the government pays for the education of children via vouchers. Parents use these vouchers to pay the school they prefer for their child. Hence, in a voucher system schools must compete for students in order to get public funding, which nicely fits our model of the regulated market. ${ }^{7}$

The paper proceeds as follows. We introduce the model in the following section. In sections 3 and 4 we consider two benchmark cases: First we analyze which curricula and which allocation of students a social planner would directly implement if he could. Then we identify the outcome in an unregulated market, i.e. in which schools compete in curricula

\footnotetext{
${ }^{7}$ Friedman (1997) makes a strong case for voucher systems in education and receives empirical support by Sandström and Bergström (2005) and Gallego (2006).
} 
and tuition fees. In section 5 we analyze equilibrium when tuition fees are regulated to a fixed level. We then compare these results and draw final conclusions from our analysis.

\section{The Model}

To keep language simple, we shall refer to all kinds of educational institutions as 'schools'. We consider a duopoly market in which schools provide education at zero costs. ${ }^{8}$ Each school $k$ decides on the level of tuition fees $T_{k} \geq 0$ and on details of the curriculum which are summarized in the variable $s_{k}$ such that a higher $s_{k}$ means that the curriculum is more challenging. A more challenging curriculum increases the productivity effect for students but makes it harder for them to meet the requirements associated with it. We measure the curriculum variable in terms of students' post-graduation income, which allows us to directly interpret $s_{k}$ as student achievement.

Teaching output spills over into the local economy, an effect we refer to as social teaching spillover and assume to be a fraction $\beta$ of aggregate student achievement in the city. Evidence for this externality abounds: For instance, Rosenthal and Strange (2008) show that the presence of workers with college education is responsible for the positive effect of agglomeration on wages. Andersson et al. (2009) report large effects of newly established higher-education institutions on output per worker in the close vicinity of the institution. We allow for some of this externality to be internalized by schools (private teaching spillover) and denote this with $\alpha \in[0, \beta]$. At times, we will restrict attention to sufficiently small $\alpha$ in order to avoid tedious case distinctions which arise when the private spillover effect becomes too dominant. To be more specific, let us denote demand for school $k$ by $D_{k}$. Then school $k$ 's profit is given by

$$
\Pi_{k}=\left(T_{k}+\alpha s_{k}\right) D_{k} .
$$

Students increase their productivity by studying, which in turn will increase their incomes after graduation. We assume that their post-graduation income will be higher the more demanding the school's curriculum is. On the downside, a more demanding curriculum $s_{k}$ at school $k$ comes at a cost $\frac{s_{k}^{2}}{2} \gamma$, where $\gamma$ is an ability parameter which is assumed to be uniformly distributed on $[\underline{\Gamma}, \bar{\Gamma}]$. Intuitively, students with higher $\gamma$ incur higher absolute and marginal costs of preparing the exam of a given curriculum. We normalize the total mass of students to unity, and we confine the analysis to $\bar{\Gamma}<2 \underline{\Gamma}$, i.e. that students are sufficiently homogenous, in order to avoid further case distinctions in the analysis. Finally, tuition fees $T_{k}$ charged by school $k$ will reduce a student's utility. Summarizing, a type- $\gamma$ student's utility from being enrolled at school $k$ is assumed to be

$$
U_{k}(\theta)=s_{k}-\frac{s_{k}^{2}}{2} \gamma-T_{k}
$$

This utility function has a number of important properties: On the one hand, it has in common with preferences in models of vertical differentiation that lower-cost students

\footnotetext{
${ }^{8}$ This is just a simplifying assumption; results would be similar with constant marginal costs. See also the discussion in the concluding section.
} 
have a higher willingness to pay for any curriculum $s_{k}$. On the other hand, there is a horizontal dimension of product differentiation as, for given prices, every student has a unique most preferred curriculum which is $s_{k}=\frac{1}{\gamma}$ and thus strictly decreasing in $\gamma$. Hence, these preferences exhibit the plausible property that a (high-cost) student may choose a less productivity-enhancing school even if it charges higher tuition fees.

A consequence of the vertical-differentiation aspect of preferences is that if a type- $\gamma$ student prefers the school with the tougher curriculum, then all students with cost types $\gamma^{\prime}<\gamma$ will do so. Hence, for given tuition fees and curricula, if both schools are able to attract any demand at all, then there will always be exactly one type of student who is indifferent between both universities, and one less able type of student who is indifferent between being enrolled at the laxer school and not being enrolled at all. In this case, these two types of student therefore fully characterize students' behavior in the market. Hence, it will prove convenient to denote the highest-cost type among students enrolled at school $k$ by $\bar{\gamma}_{k}$.

With this definition, and without loss of generality referring to the school with the more challenging curriculum as school 1 (i.e., $s_{1}>s_{2}$ ), we can now set up schools' profit functions. As students differ only in their costs of preparing exams, the average student achievement of school $k$ is simply $s_{k}$. Hence, schools' profits are given by

$$
\begin{aligned}
& \Pi_{1}=\left(T_{1}+\alpha s_{1}\right) \frac{\bar{\gamma}_{1}-\underline{\Gamma}}{\bar{\Gamma}-\underline{\Gamma}} \\
& \Pi_{2}=\left(T_{2}+\alpha s_{2}\right) \frac{\bar{\gamma}_{2}-\bar{\gamma}_{1}}{\bar{\Gamma}-\underline{\Gamma}},
\end{aligned}
$$

provided that there are some students who enrol at all at the respective school (i.e., $\underline{\Gamma}<$ $\left.\bar{\gamma}_{1}<\bar{\gamma}_{2} \leq \bar{\Gamma}\right)$.

By contrast, a benevolent social planner would seek to maximize the sum of students' aggregated utility and teaching spillover:

$$
\max _{s_{1}, s_{2}, \underline{\theta}_{1}, \underline{\theta}_{2}}(1+\beta)\left[s_{1} \frac{\bar{\gamma}_{1}-\underline{\Gamma}}{\bar{\Gamma}-\underline{\Gamma}}+s_{2} \frac{\bar{\gamma}_{2}-\bar{\gamma}_{1}}{\bar{\Gamma}-\underline{\Gamma}}\right]-\frac{s_{1}^{2}}{2} \frac{\bar{\gamma}_{1}^{2}-\underline{\Gamma}^{2}}{2(\bar{\Gamma}-\underline{\Gamma})}-\frac{s_{2}^{2}}{2} \frac{\bar{\gamma}_{2}^{2}-\bar{\gamma}_{1}^{2}}{2(\bar{\Gamma}-\underline{\Gamma})}
$$

subject to $\underline{\Gamma} \leq \bar{\gamma}_{1} \leq \bar{\gamma}_{2} \leq \bar{\Gamma}$. The first summand in the social planner's objective function is the total social benefit from aggregate student achievement in both schools, which is just the sum of students' aggregate post-graduation income and the social teaching spillover. The second and third summands are average cost of preparing the exams at each school. Comparing the objective functions (3), (4) and (5) shows that, irrespectively of the market structure, there are two potential kinds of externality: First, the already discussed fact that schools may reflect only part of the teaching spillover whenever $\alpha<\beta$, and second, the fact that schools care only about their decisions' influence on the marginal students, whereas the social planner cares about the average student.

In line with the theoretical literature on vertical differentiation, we sought to keep the model tractable by assuming distributions and functional forms which may seem very special to the general reader. However, we shall argue in the conclusions that the key effects identified 
in this paper will hold for a large variety of functional forms. What really drives the main difference of our results compared to existing theories, the additional inefficiency of regulated tuition fees identified in Proposition 4, is what we believe is a distinctive feature of the choice of curricula: It is the implicit assumption that less able students will prefer laxer curricula even if tuition fees are constant.

\section{Efficient Curricula}

As a benchmark, let us analyze how a social planner would locate the two schools on the range of curricula and allocate the potential students between these schools. Loosely speaking, the social planner trades off a higher number of students creating a positive social surplus when enrolling at a school if the curriculum is lax against a higher social surplus created by students already enrolled when it is tough.

Taking the partial first derivatives of (5) w.r.t. the curricula $s_{1}, s_{2}$ gives us the first-order conditions

$$
\begin{aligned}
& s_{1}=\frac{2(1+\beta)}{\bar{\gamma}_{1}+\underline{\Gamma}} \\
& s_{2}=\frac{2(1+\beta)}{\bar{\gamma}_{2}+\bar{\gamma}_{1}}
\end{aligned}
$$

These equations show that, a higher social teaching spillover $(\beta)$ makes society prefer tougher curricula for a given allocation of students between schools.

Turning to the efficient allocation of students between schools, note that due to students' heterogeneity it can never be optimal to have only one active school, i.e. $\underline{\Gamma}<\bar{\gamma}_{1}<\bar{\gamma}_{2}$ will be satisfied by the simple first-order conditions anyway and can therefore be ignored. Hence, the only relevant constraint is $\bar{\gamma}_{2} \leq \bar{\Gamma}$. The following Proposition shows that this constraint is indeed binding:

Proposition 1. The socially optimal sets of students to be enrolled at each school, and socially optimal curricula are

$$
\begin{aligned}
\bar{\gamma}_{1}^{f} & =\sqrt{\bar{\Gamma} \underline{\Gamma}} \\
\bar{\gamma}_{2}^{f} & =\bar{\Gamma} \\
s_{1}^{f} & =\frac{2(1+\beta)}{\sqrt{\underline{\Gamma}}(\sqrt{\bar{\Gamma}}+\sqrt{\underline{\Gamma}})} \\
s_{2}^{f} & =\frac{2(1+\beta)}{\sqrt{\bar{\Gamma}}(\sqrt{\bar{\Gamma}}+\sqrt{\underline{\Gamma}})} .
\end{aligned}
$$

Proof. See the Appendix.

The efficient set of students who should be enrolled at all (which is the entire population in our model), and the efficient allocation thereof between schools are independent of the teaching spillover. 


\section{The Unregulated Market Outcome}

If there is no regulation, then schools will choose curricula and tuition fees so as to maximize their profits (1). We follow the literature on product differentiation by assuming that tuition fees are easier to change on short notice than curricula. We reflect this assumption by modeling the game in a two-stage manner: In the first stage, schools choose curricula simultaneously, and in the second stage they choose tuition fees simultaneously. We solve the game via backwards induction.

Suppose without loss of generality that school 1 has chosen a tougher curriculum in stage 1, i.e. $s_{1}>s_{2}$. Then, due to the monotonicity of students' preferences with respect to $\gamma$, school 1 will attract low-cost students even if it charges higher tuition fees than school 2 . More specifically, there is exactly one type of student $\bar{\gamma}_{1}$ indifferent between both schools, given by $s_{1}-\frac{s_{1}^{2}}{2} \bar{\gamma}_{1}-T_{1}=s_{2}-\frac{s_{2}^{2}}{2} \bar{\gamma}_{1}-T_{2}$, which is equivalent to

$$
\bar{\gamma}_{1}=\frac{2}{s_{1}+s_{2}}\left(1-\frac{T_{1}-T_{2}}{s_{1}-s_{2}}\right) .
$$

Students prefer school 1 over school 2 if and only if their cost type is $\gamma \leq \bar{\gamma}_{1}$.

Furthermore, there may be students who choose not to be enrolled at all. However, we assume for the moment that all students choose to enrol, which is equivalent to $s_{2}-\frac{s_{2}^{2}}{2} \bar{\Gamma}-T_{2} \geq$ 0 , and which will be proven in Proposition 2 to be satisfied in equilibrium. Under this assumption $\bar{\gamma}_{2}=\bar{\Gamma}$. Using (12) to substitute for $\bar{\gamma}_{1}$ in the schools' profit functions (3) and (4), taking their derivatives w.r.t. $T_{i}$, solving the resulting system of two equations and substituting for the equilibrium choices thereby obtained yields schools' equilibrium profits in the pricing stage presented in the following Lemma:

Lemma 1. Suppose that in the first stage curricula $s_{1}>s_{2}$ have been chosen such that in the equilibrium of the second-stage subgame (i) both schools will charge strictly positive tuition fees and (ii) even the least able student $\bar{\Gamma}$ will strictly prefer being enrolled at school 2 to not being enrolled at all. Then, profits earned by schools in equilibrium are

$$
\begin{aligned}
\Pi_{1}^{*} & =\frac{2\left(s_{1}-s_{2}\right)}{9\left(s_{1}+s_{2}\right)(\bar{\Gamma}-\underline{\Gamma})}\left((1+\alpha)-\frac{s_{1}+s_{2}}{2}(2 \underline{\Gamma}-\bar{\Gamma})\right)^{2} \\
\Pi_{2}^{*} & =\frac{2\left(s_{1}-s_{2}\right)}{9\left(s_{1}+s_{2}\right)(\bar{\Gamma}-\underline{\Gamma})}\left(\frac{s_{1}+s_{2}}{2}(2 \bar{\Gamma}-\underline{\Gamma})-(1+\alpha)\right)^{2} .
\end{aligned}
$$

Proof. See the Appendix.

The area in the $s_{1}-s_{2}$ space for which (13) and (14) are the schools' relevant objective functions in the first stage is thus bounded by the following conditions: First, if curricula are so similar that price competition makes schools charge zero tuition fees and rely solely on the private teaching spillover, small changes in the curricula will not change equilibrium tuition fees, so that school's equilibrium profit functions will be different from (13) and (14). Second, as school 1's curriculum gets tougher, it becomes less attractive for many of the 
higher-cost students, so that school 2 can afford to charge high tuition fees. This, however makes school 2 less attractive to the highest-cost types of students. To avoid loosing them, school 2 will leave its tuition fees constant in some interval of school 1's curriculum. By contrast, (13) takes into account an effect of school 1's curriculum on school 2's equilibrium tuition fees. Hence, the objective functions in the Lemma are no longer valid in this area.

With Lemma 1 it is easy to derive equilibrium curricula in the first stage of the game if curricula are restricted to the set in which Lemma 1 is valid. The following proposition characterizes this equilibrium of the restricted game and derives a sufficient condition which guarantees that the equilibrium of the unrestricted game is equivalent to it.

Proposition 2. Suppose that tuition fees are unregulated and $\alpha$ is sufficiently low. There is a constant $\lambda \in(1,2)$ which is independent of all other parameters in our model such that

(i) If $\bar{\Gamma}<\lambda \underline{\Gamma}$, all students are enrolled, and equilibrium curricula are

$$
\begin{aligned}
s_{1}^{e} & =\frac{2(1+\alpha)}{\sqrt{2 \underline{\Gamma}-\bar{\Gamma}}(\sqrt{2 \bar{\Gamma}-\underline{\Gamma}}+\sqrt{2 \underline{\Gamma}-\bar{\Gamma}})} \\
s_{2}^{e} & =\frac{2(1+\alpha)}{\sqrt{2 \bar{\Gamma}-\underline{\Gamma}}(\sqrt{2 \bar{\Gamma}-\underline{\Gamma}}+\sqrt{2 \underline{\Gamma}-\bar{\Gamma}})},
\end{aligned}
$$

(ii) If $\bar{\Gamma} \geq \lambda \underline{\Gamma}$, then equilibrium is at a boundary solution such that the highest-cost student $\bar{\Gamma}$ is just indifferent between being enrolled at school 2 and not being enrolled at all.

(iii) In both cases, both schools' equilibrium curricula are strictly increasing in $\alpha$. The tougher school's equilibrium curriculum is tougher than the efficient one for a sufficiently close to $\beta$, and the laxer school's curriculum is always laxer than the efficient one.

(iv) Given the schools' equilibrium choices of curriculum in part (i), the fraction of students going to the tough school is inefficiently large as long as $\alpha$ is sufficiently close to $\beta$. The same would hold even if curriculum choices were efficient.

Proof. See the Appendix.

If students are rather homogenous, there is not much room for schools to differentiate their curricula in order to relax price competition. Hence, all students participate and earn positive surplus..$^{9}$ Hence, equilibria in part (i) of Proposition 2 are calculated without incorporating any effect of curricula on the highest-cost student's participation decision. As students

\footnotetext{
${ }^{9}$ Note that Proposition 2 requires the schools' private benefit $\alpha$ from teaching spillover to be sufficiently low. If it was too dominant, the equilibrium set out in part (i) may be destroyed by the lax schools' incentives to move to a curriculum in which the tough school charges zero tuition fees. We chose to analyze only the former case, as we expect schools' private benefits to be rather unimportant as compared to the social benefit of teaching spillover.
} 
become more heterogenous, this gives schools a fast-growing incentive to differentiate. For instance, if it was for the $s_{1}^{e}$ defined in (15), school 1 would like to choose an infinitely tough curriculum as $\bar{\Gamma}$ approaches $2 \underline{\Gamma}$. However, this is not feasible: As differentiation relaxes competition to such an extent that tuition fees are sufficiently high, the highest-cost student's participation constraint binds, which will affect the laxer school's behavior in the pricing stage: Its price decision is now restricted by the requirement not to loose the highestcost student, so that it does not pay off any more for the tougher school to further relax competition by making its curriculum tougher. Hence, for heterogenous students there is a boundary solution given by the highest-cost student's participation constraint.

For both the interior and the boundary solutions, equilibrium curricula are increasing in the schools' private teaching spillover parameter. Furthermore, absent any teaching spillover $(\alpha=\beta=0)$ equilibrium curricula are excessively differentiated, i.e. the tough school's curriculum is excessively tough and the lax school's excessively lax. This confirms the wellknown result from the IO literature that that firms tend to differentiate excessively in order to relax price competition. ${ }^{10}$ However, the externality resulting from the teaching spillover has a countervailing effect on the inefficiency of the tough school's curriculum: As the efficient curricula are also increasing in $\beta$, it may be possible for large $\beta$ and small $\alpha$ that the tough school's curriculum is efficient or even insufficiently tough. Note that this does not mean that the externality will have a beneficial effect here, as the lax school's curriculum will be even more inefficient in such a case.

Part (iv) of Proposition 2 shows that even if we take schools' choices of curricula as given, a social planner would order less students to go to the tough school than what actually happens in equilibrium. Hence, the pricing stage introduces another inefficiency. Note that even if the condition that $\alpha$ be close to $\beta$ is not satisfied, in which case the inefficiency of equilibrium allocation of students may go in either direction, it is clear that this allocation can be efficient only by a mere fluke.

Summing up, we have two sources for inefficiency in the unregulated market: First, it is only the private instead of the social benefit of teaching spillover which enters the schools' objective functions. Second, the market suffers from the well-known inefficiencies, excessive differentiation and oligopoly pricing.

\section{Fixed Tuition Fees}

Assume now that tuition fees students must pay at both schools are regulated to $T \geq$ 0 . Then the individual benefit of product differentiation for schools that it reduces price competition no longer holds, as prices are given anyway. To see this, recall equation (12), which describes the allocation of students among the two schools, provided that both schools can attract some student. As the difference in schools' tuition fees is zero, this allocation depends only on the schools' curricula. More specifically, a type- $\gamma$ student prefers school 1 over school 2 if and only if his most preferred curriculum $\frac{1}{\gamma}$ is tougher than the schools'

\footnotetext{
${ }^{10}$ See, for instance, Shaked and Sutton (1982).
} 
average curriculum. The following Lemma shows that without the motive of relaxing price competition, schools do not have any incentive to differentiate their curricula.

Lemma 2. There cannot exist a pure strategy equilibrium in which schools set different curricula.

Proof. See the Appendix.

In the proof of Lemma 2 we show that in any situation in which schools offer different curricula, at least one school will have an incentive to move its curriculum closer to the rival school. For instance, if all students strictly prefer being enrolled in some school to not studying at all, the laxer school can always increase its profit by offering a slightly tougher curriculum: The lax school's externalities from student achievement increase for every student enrolled at the lax school, and the lax school's student body increases as the average curriculum gets tougher.

Lemma 2 established that if a pure strategy equilibrium exists, then both schools must choose the same curriculum in equilibrium. To assess whether such an equilibrium exists and characterize it, assume now that there exists such a symmetric pure-strategy equilibrium. Let $s^{*}$ denote the equilibrium choice of curriculum for each university, which can be the case only if it is not profitable for a school to undercut or overbid $s^{*}$. Recall that $s^{*}$ is the most preferred curriculum of type $\gamma^{*}:=\frac{1}{s^{*}}$. Hence, unilaterally slightly undercutting (overbidding) $s^{*}$ would give school $i$ all students enrolled with cost parameters below (above) $\gamma^{*}$ instead of half the total demand. As its teaching spillover remains almost unchanged after such a slight deviation, a slightly deviating school cares only about total demand. Hence, such a marginal deviation is unprofitable if and only if there are exactly half of all enrolled students below and above $\gamma^{*}$, respectively. The following Proposition derives the unique curriculum satisfying this requirement: ${ }^{11}$

Proposition 3. If tuition fees are exogenously fixed at $T$, there is a unique pure-strategy equilibrium with both schools offering identical curricula $s_{i}^{*}=s_{j}^{*}=s^{*}$, which is given by

$$
s^{*}=\max \left\{\frac{2}{\bar{\Gamma}+\underline{\Gamma}}, \sqrt{\frac{2 T}{\underline{\Gamma}}}\right\}
$$

whenever $T<\frac{1}{2 \underline{\Gamma}}$. The total number of enrolled students is $\frac{2}{\bar{\Gamma}-\underline{\Gamma}}\left(\frac{1}{s^{*}}-\underline{\Gamma}\right)$.

Proof. See the Appendix.

A striking implication of proposition 3 is that the teaching spillover does not influence the schools' decisions on curricula at all. Instead, schools' behavior is equivalent to demand maximization: For a slight deviation from the rival's curriculum, the change in teaching

\footnotetext{
${ }^{11}$ Of course, the proof must, and does, include also an analysis of large deviations from $s^{*}$.
} 
spillover is marginal, whereas demand is discontinuous at this point for almost all curricula the rival may set.

If $T \leq \frac{2 \Gamma}{(\bar{\Gamma}+\Gamma)^{2}}$, all students prefer being enrolled at a university offering curriculum $s^{*}=$ $\frac{2}{\bar{\Gamma}+\Gamma}$ to not being enrolled ('full market coverage'), so that type $\gamma^{*}=\frac{1}{s^{*}}$ is obviously the median student. For $\frac{2 \underline{\Gamma}}{(\bar{\Gamma}+\underline{\Gamma})^{2}}<T<\frac{1}{2 \underline{\Gamma}}$, the highest-cost type $\bar{\Gamma}$ is better off not being enrolled if the curriculum $\frac{2}{\bar{\Gamma}+\underline{\Gamma}}$ is offered. Hence, the equilibrium in this case is the curriculum which is most preferred by the median among all enrolled students. Under curriculum $s^{*}=\sqrt{\frac{2 T}{\Gamma}}$, the type indifferent between being enrolled and not being enrolled is $\bar{\gamma}=\frac{2}{s^{*}}-\underline{\Gamma}$, so that the median among all enrolled students is indeed $\gamma^{*}=\frac{1}{s^{*}}$. Furthermore, in this case, higher fixed tuition fees make, for given curricula, schools less attractive for the highest-cost students, thus increasing average ability. Hence, the equilibrium curriculum, which lies in the center of the enrolled students' most preferred curricula, gets tougher. It follows that both the median student's $\left(\gamma^{*}=\frac{1}{s^{*}}\right)$ and the marginal student's $\left(\bar{\gamma}=\frac{2}{s^{*}}-\underline{\Gamma}\right)$ cost types decline as the regulated tuition fee increases. ${ }^{12}$

Proposition 3 shows that the level of the fixed tuition fee $T$ plays an important role in determining the equilibrium. Every $T$ induces a unique equilibrium curriculum given by equation (17) and thus a unique set of participating students. This means that, from a welfare point of view, the regulator's choice of $T$ in fact boils down to choosing a pair of equilibrium curriculum $s^{*} \in\left[\frac{2}{\bar{\Gamma}+\underline{\underline{\Gamma}}}, \frac{1}{\bar{\Gamma}}\right]$ and set of enrolled students $\left[\underline{\Gamma}, \frac{2}{s^{*}}-\underline{\Gamma}\right]$. Given this relationship of $T$ and the equilibrium choices, it will be interesting to find the best tuition fee a regulator can set, given that he chooses to regulate tuition fees at all. There are two obvious inefficiencies associated with fixed tuition fees: First, equilibrium curricula is always symmetric, whereas the students' heterogeneity calls for some product differentiation. Second, there is only one regulatory instrument, the fixed level of tuition fees $T$, which is supposed to regulate two equilibrium outcomes at the same time, the choice of curriculum and the set of enrolled students.

In order to separate these effects, let us first derive the socially optimal curriculum and set of students given that schools are homogenous. In this case, the regulator's problem is to

$$
\max _{s, \bar{\gamma}}(1+\beta) s \frac{\bar{\gamma}-\underline{\Gamma}}{\bar{\Gamma}-\underline{\Gamma}}-\frac{s^{2}}{2} \frac{\bar{\gamma}^{2}-\underline{\Gamma}^{2}}{2(\bar{\Gamma}-\underline{\Gamma})}
$$

subject to $\bar{\gamma} \in[\underline{\Gamma}, \bar{\Gamma}]$. Taking the first derivative w.r.t. $s$ yields the first-order condition

$$
s=\frac{2(1+\beta)}{\bar{\gamma}+\underline{\Gamma}}
$$

which maximizes the regulator's objective function for given $\bar{\gamma}$. The following Lemma shows that the regulator's optimal choice of the set of enrolled students is a boundary solution:

\footnotetext{
${ }^{12}$ Of course, an equilibrium cannot exist if $T \geq \frac{1}{2 \underline{\Gamma}}$, as even the lowest-cost student's $(\gamma=\underline{\Gamma})$ net surplus from education can get as large as that.
} 
Lemma 3. If the regulator could force schools to choose a certain curriculum s and students to enrol or not, with the only restriction being that schools' curricula are homogenous, the socially optimal curriculum is $s=\frac{2(1+\beta)}{\bar{\Gamma}+\underline{\Gamma}}$, and all students should be enrolled independently of $\beta$.

Proof. See the Appendix.

As one would expect, a higher social teaching spillover calls for tougher curricula. Furthermore, Lemma 3 illustrates the aforementioned problem associated with controlling two equilibrium choices with just one regulative instrument: Inducing full participation of students in equilibrium is associated with the curriculum $s=\frac{2}{\bar{\Gamma}+\underline{\Gamma}}$, which is inefficiently lax. On the other hand, if the social planner induces schools to set up the efficient curriculum (given an homogenous equilibrium), students' participation will be inefficiently low.

This inevitable inefficiency is surprising at first glance, as one might conjecture that the optimal regulation is trivially $T=0$, which seems to align private and social costs and benefits. To see this, suppose that $\alpha=\beta=0$. A social planner would then seek to maximize just the sum of student achievement less students' expected effort costs. To achieve this, he must (i) order schools to choose the 'central' curriculum, which minimizes the average distance from the enrolled students' most preferred curricula, and (ii) make sure that the marginal student's productivity is equal to his effort cost under this curriculum. As we know from Proposition 3, the equilibrium curriculum will, for every $T$, always be that curriculum which is most preferred by the median enrolled student. Hence, the social planner need care only about balancing the marginal student's productivity and effort cost, which is clearly achieved if and only if $T=0$. This reasoning is, however, no longer valid if $\beta>0$. Even if the entire social teaching spillover was internalized by schools $(\alpha=\beta)$, the teaching spillover works, in fact, like an externality, as schools are forced by the mechanics of equilibrium to ignore it when choosing their curricula. Hence, simply setting the non-distorting tuition fee $T=0$ will not align individual and social interests in this case.

Given this trade-off between inducing schools to offer a tougher curriculum in order to account for the teaching spillover, and losing some students that should be educated, a natural question is how a social planner should balance these inefficiencies. The following Proposition establishes that the 'participation effect' strictly outweighs the 'teaching spillover effect' whenever $\beta>0$, so that the optimal regulation yields full student participation:

Proposition 4. (i) The (second-best) optimal regulation of tuition fees induces schools to choose the curriculum $s^{*}=\frac{2}{\bar{\Gamma}+\underline{\underline{\Gamma}}}$, under which all students will be enrolled in equilibrium.

(ii) Curricula are always too lax under the second-best regulation.

Proof. See the Appendix.

Proposition 4 implies that the optimal regulation will induce schools to choose inefficiently lax curricula whenever $\beta>0$. Hence, the anecdotal concern that all universities may excessively reduce the requirements for a degree in order to please low-ability students is 
confirmed, but in our model this incentive arises in the regulated case, whereas in the public discussion this problem is often attributed to an unregulated market.

Even more strikingly, the equilibrium curriculum offered by both schools under the optimal regulation does not depend at all on the teaching spillover, neither on the social nor on schools' private one. Hence, the regulator's attempt to influence schools' choices of curriculum via regulated tuition fees comes at two kinds of cost: Any differentiation of curricula is removed, and so is any incentive for schools to take the teaching spillover into account. Whether the benefits of regulation exceed the cost depend on the extent of market imperfection in the unregulated case. This will be discussed in more detail in the next section.

\section{Comparison}

The analysis in sections 4 and 5 suggests that a comparison between the two regimes will depend on three model parameters: First, the social teaching spillover $\beta$ determines how the equilibrium outcomes in the two regimes are valuated in the social welfare account. As the teaching spillover is defined to relate only to student achievement, larger $\beta$ will make that regime comparatively more attractive which results in higher average student achievement. This is good news for the practical application of our theory, as it implies that the more severe externality problem is, the more important for the regulation decision is average student achievement, which is much easier to measure than students' average cost of preparing exams.

Second, schools' private teaching spillover $\alpha$ determines the extent of inefficiency in the unregulated market, but does not affect equilibrium at all if tuition fees are regulated. Hence, larger $\alpha$ will make the unregulated market comparatively more attractive.

Last, the degree of heterogeneity of students' types plays an ambiguous role: If students are homogenous, then the lack of differentiation of curricula under regulated tuition fees is less harmful, but at the same time the problem of excessive differentiation in the unregulated market is less pronounced. Which one of the two effects dominates will crucially depend on the details of the distribution of students' types and is therefore an empirical issue.

The impact of teaching spillover can be nicely illustrated in the following numerical example, for which equilibrium outcomes happen to be easy to calculate.

Example 1. Let $\bar{\Gamma}=\frac{3}{2} \underline{\Gamma}$. As $\frac{3}{2}<\lambda$, equilibrium curricula in the unregulated market are given by the interior solution (15) and (16). Hence, $s_{1}^{e}=\frac{4(1+\alpha)}{3 \underline{\Gamma}}$, and $\bar{\gamma}_{1}=\frac{7}{6} \underline{\Gamma}$. With (5), this yields social welfare in the unregulated market,

$$
W^{u}=(1+\beta)(1+\alpha) \frac{8}{9 \underline{\Gamma}}-(1+\alpha)^{2} \frac{14}{27 \underline{\Gamma}} .
$$

Under the optimal regulation of tuition fees, $s=\frac{4}{5 \Gamma}$, so that (18) implies that

$$
W^{r}=\frac{2(1+2 \beta)}{5 \underline{\Gamma}} .
$$

We can thus easily analyze the impact of the teaching spillover: 
- Absent any teaching spillover $(\alpha=\beta=0)$ regulated tuition fees would yield a higher welfare than an unregulated market.

- However, a positive social spillover $\beta>0$ reduces this advantage even if it is not internalized at all $(\alpha=0)$, as average student achievement in the unregulated market, $\frac{8}{9 \underline{\Gamma}}$, is larger than that under regulated tuition fees (which is equal to $s=\frac{4}{5 \underline{\Gamma}}$ ).

- This effect is even stronger if the private teaching spillover is varied together with the social teaching spillover (for instance, if $\alpha$ is a fixed fraction of $\beta$ ).

\section{Conclusions and Discussions}

We have investigated the effects of regulating competition between providers of higher and secondary education and found that it is regulated tuition fees which induce universities to set up excessively lax curricula. This is in sharp contrast to the standard argument discussed in the public, who attribute this inefficiency to an unregulated market. This inefficiency is increasing in the size of the positive externality of student achievement for the local economy. Furthermore, even if some of this externality can be appropriated by schools, it will not influence equilibrium. On the other hand, the unregulated market features equilibrium curricula which are excessively differentiated and increasingly tough in schools' private benefits from teaching spillover.

Our analysis of the regulated case rests on the assumption that schools choose curricula autonomously because close teacher supervision in the classroom is almost impossible. If curricula were able to be regulated directly, inefficiencies would not arise because schools would then simply be ordered to offer efficient curricula. Of course regulators would like to try to get a grip on the content in particular in the context of regulated tuition fees when curricula are too lax and too uniform. Germany's higher education sector provides an interesting example in this context. Tuition fees are low and very regulated and policy makers are busy ensuring minimum standards for curricula via a tough accreditation process for degree programs. Moreover, tertiary education is separated between more academic institutions (research universities) and applied institutions (universities of applied sciences). The latter universities must hire professors with sufficient business or other practical experiences to make sure that teaching does not become too academic. This effort may be interpreted as the regulator's attempt to impose a larger separation in curricula between these different types of universities.

The aim of our analysis was to examine in which regulatory environment the suspected incentive to be excessively lax on study requirements is more pronounced. In doing so, we focused almost exclusively on the choice of curriculum and disregarded other arguments which are often brought forward in defense of regulation, such as capital market imperfections. Hence, we had to be careful when interpreting our comparison of the two regimes. In particular, we are well aware that our comparing discussions in Section 6 do not prove that the unregulated market was always superior, for instance, for a sufficiently large private benefits to schools from teaching spillover ( $\alpha$ in our model). We rather see this comparison 
as an example which shows that the countervailing effect of regulation we have identified may be sufficiently severe as to make up for certain positive effects of regulation.

At the same time, we believe that our basic insight that the inefficiency associated with regulated tuition fees is more severe for a higher social teaching spillover is rather robust: The result that the inefficiency of equilibrium curricula under the optimal regulation is increasing in $\beta$ is a direct consequence of schools' behavior of demand maximization, which in turn follows from the plausible assumption that weak students prefer easy ways of getting a degree for given tuition fees. In the case of the unregulated market, equilibrium curricula do respond to changes in schools' private benefits of teaching spillover and, if these private benefits are somehow linked to social benefits, also to changes in social benefit of teaching spillover. Hence, although our assumptions may seem special, all we need for our results are curricula as characteristics of schools, together with well-known and robust features of models of vertical product differentiation.

Having said this, we note that it is possible to extend our model in multiple ways in order to achieve comparability to results of previous studies. In the following we shall discuss briefly the consequences for our results of some of these extensions.

Costly teaching. If teaching is costly, both society and schools prefer some of the highest-cost students not to study any more. Hence, in the regulated case the optimal regulation will entail a positive fixed tuition fee. We considered a similar model where teaching entails a fixed cost $C$ per student, but it turned out that the ranking of the unregulated market and the optimal regulation remained unaffected for $\alpha=0$, and so did the comparative statics with respect to $\alpha$. The intuitive reason is that schools' and society's interests are aligned concerning the effects of tuition costs.

Heterogeneous schools. In reality, differences in the teaching spillover exist not only between institutions which are active on different market, such as secondary and higher education, but also within markets. Hence, the natural question arises how our results would change in a model where schools have heterogeneous $\alpha_{k}$ and $\beta_{k}$. It turns out that equilibrium curricula under regulated tuition fees are still homogenous (Proposition 3), which follows directly from the fact that schools' best replies are independent of $\alpha_{k}$. Hence, what changes is just that the social preference for differentiation increases, which makes regulated tuition fees even less attractive.

Distribution of students' types. The uniform distribution of types seems rather strong, but our qualitative results are robust to moderate deviations from this assumption. We analyzed the model with different assumptions on the distribution of types, and as long as the distribution of types is sufficiently smooth, the optimal deviation from the competitor's curriculum in the regulated case is still the minimum deviation. Hence, in this case, the result that under regulated tuition fees the equilibrium curriculum is where exactly half of the enrolled students would prefer a tougher and half of them would prefer a laxer curriculum goes through. If, however, the density function of types exhibits steep peaks, it may be the case that attracting the group of students represented by such a peak dominates all other 
effects. In this case, an equilibrium with heterogeneous curricula might result even in the case of regulated tuition fees.

Fixed heterogenous tuition fees. In our model, fixed tuition fees are associated with large inefficiencies because they lead to a symmetric equilibrium in curricula. One could, therefore, conjecture that heterogeneous fixed fees may improve things. Indeed, in Kemnitz (2007) such a regulation even implements the first best if these fixed tuition fees are set optimally. In our model, however, the horizontal-differentiation character of the students' preferences induces the "cheaper" school to steal its rival's students whenever the demand for the expensive school is positive. Intuitively, the high-fee school will attract zero demand whenever its curriculum is too similar to that of the low-fee school. However, if the high-fee school seeks to escape this by setting a very demanding or a very lax curriculum, the low-fee school's best reply to this is again to move closer to the high-fee school, thereby driving the demand for the high-fee school again down to zero. Hence, there is a pure strategy equilibrium only if the low-fee school can keep the high-fee school out of the market for any choice of curriculum of the high-fee school. Otherwise, the equilibrium will be in mixed strategies, which makes it impossible to implement a given set of curricula with certainty.

Vouchers. We have assumed for simplicity that schools receive exactly the tuition fees their students pay. In the public discussion it is sometimes suggested that skilled graduates pay higher taxes to the government, and per-student compensations for educational institutions should be independent from those taxes. Note that such an assumption would not make any difference in our model, as schools maximize demand in equilibrium and do not care about the exact size of per-student revenue at all.

Peer-group effects. Unlike many contributions our paper does not consider peer effects between students which are prominent in Kemnitz (2007), Epple et al. (2006), Lazear (2001) and empirical papers like Hoxby (2000). Peer effects are a type of network effects and imply the impact of a student on his fellow students. In theoretical models, they usually are captured by the average ability of students in a school (like in Epple et al. (2006) or Kemnitz (2007)). In this case it is typically efficient to enrol students into different schools according to their ability. Note that Meier (2004) provides an exception to this rule of thumb. Full competition between schools typically achieves this selection. On the other hand Hoxby (2000) argues that good students provide support in particular for bad students such that competition leads to allocations which are beneficial for the good students but harmful for the overall welfare. Econometric problems are a key cause for this heterogeneity, as the selfselection of students into peer groups typically precludes the estimation of counterfactual evidence. ${ }^{13}$ Furthermore teachers and other factors may shape the peer effects. Hence it is not surprising that Epple et al. (2006) refer to all types of empirical evidence for peer effects.

Our main results do not change qualitatively if we model peer effects indirectly by means of a positive impact of the students' average cost type on a school's profit (as for instance in

\footnotetext{
${ }^{13}$ See Eisenkopf (2010).
} 
Kemnitz (2007)). In this case, under regulated tuition fees schools will reflect the beneficial effect of stricter curricula on average student type and thus on their profits, so that equilibrium curricula will be stricter than in the absence of peer-group effects, and less students will enrol. The results that there are only symmetric equilibria, that equilibrium curricula are independent of the teaching spillover and therefore the inefficiency associated with regulated tuition fees is increasing in this spillover will, however, continue to hold.

\section{Appendix}

\section{Appendix A. Proof of Proposition 1}

Recalling that the only relevant constraint is $\bar{\gamma}_{2} \leq \bar{\Gamma}$, the Kuhn-Tucker conditions are (6), (7) and

$$
\begin{aligned}
\bar{\gamma}_{1} & =\frac{2(1+\beta)}{s_{1}+s_{2}} \\
\frac{s_{2}}{\bar{\Gamma}-\underline{\Gamma}\left(1+\beta-\frac{s_{2} \bar{\gamma}_{2}}{2}\right)-\lambda} & =0 \\
\lambda\left(\bar{\gamma}_{2}-\bar{\Gamma}\right) & =0 \\
\lambda & \geq 0 .
\end{aligned}
$$

Using (7) to substitute for $s_{2}$ in (A.2) yields

$$
\lambda=\frac{2(1+\beta)^{2}}{\bar{\Gamma}-\underline{\Gamma}} \frac{\bar{\gamma}_{1}}{\bar{\gamma}_{2}+\bar{\gamma}_{1}}>0,
$$

which proves that the constraint is binding, i.e. $\bar{\gamma}_{2}=\bar{\Gamma}$.

Hence, the socially efficient curricula and allocations of students can be obtained by solving the system of equations (6), (7) and (A.1) when substituting for $\bar{\gamma}_{2}=\bar{\Gamma}$. Using (6) and (7) to substitute for $s_{1}$ and $s_{2}$ in (A.1) yields

$$
\bar{\gamma}_{1}=\frac{\left(\bar{\gamma}_{1}+\underline{\Gamma}\right)\left(\bar{\gamma}_{1}+\bar{\Gamma}\right)}{2 \bar{\gamma}_{1}+\underline{\Gamma}+\bar{\Gamma}},
$$

which is equivalent to (8). Using this to substitute for $\bar{\gamma}_{1}$ in (6) and (7) then yields the efficient curricula (10) and (11).

\section{Appendix B. Proof of Lemma 1}

Every student may choose among three options: Being enrolled at school 1, at school 2, and not being enrolled at all. Note first that students' types differ only in how they valuate curricula, and not tuition fees. Hence, for any combination of tuition fees, if there are some types who prefer the tough school 1 and some who prefer the lax school 2, then there will be exactly one type $\bar{\gamma}_{1}$ given by equation (12) such that a student prefers school 1 over school 
2 if and only if $\gamma<\bar{\gamma}_{1}$. Similarly, if there is some type which prefers school 2 and some who prefer not being enrolled at all, then there will be exactly one type

$$
\bar{\gamma}_{2}=\frac{2}{s_{2}}\left(1-\frac{T_{2}}{s_{2}}\right)
$$

such that a student prefers school 2 over not being enrolled if and only if $\gamma<\bar{\gamma}_{2}$. In that case, $\bar{\gamma}_{1}<\bar{\gamma}_{2}$.

Suppose for the moment that both schools attract a strictly positive number of students, which will turn out to be the case in equilibrium. Then, for any given pair of curricula $s_{1}>s_{2}$ chosen in the first stage, the schools' profits are

$$
\begin{aligned}
& \Pi_{1}\left(T_{1} ; T_{2}, s_{1}, s_{2}\right)=\left(T_{1}+\alpha s_{1}\right) \frac{\frac{2}{s_{1}+s_{2}}\left(1-\frac{T_{1}-T_{2}}{s_{1}-s_{2}}\right)-\underline{\Gamma}}{\bar{\Gamma}-\underline{\Gamma}} \\
& \Pi_{2}\left(T_{2} ; T_{1}, s_{1}, s_{2}\right)= \begin{cases}\left(T_{2}+\alpha s_{2}\right) \frac{\bar{\Gamma}-\frac{2}{s_{1}+s_{2}}\left(1-\frac{T_{1}-T_{2}}{s_{1}-s_{2}}\right)}{\bar{\Gamma}-\frac{\Gamma}{s_{2}},}, & \text { if } T_{2} \leq s_{2}\left(1-\frac{s_{2}}{2} \bar{\Gamma}\right) ; \\
\left(T_{2}+\alpha s_{2}\right) \frac{\frac{2}{s_{2}}\left(1-\frac{T_{2}}{s_{2}}\right)-\frac{2}{s_{1}+s_{2}}\left(1-\frac{T_{1}-T_{2}}{s_{1}-s_{2}}\right)}{\bar{\Gamma}-\underline{\Gamma}}, & \text { otherwise. }\end{cases}
\end{aligned}
$$

Taking the derivative of $\Pi_{1}$ w.r.t. $T_{1}$, and recalling that negative tuition fees are ruled out, yields school 1's best-reply curve:

$$
T_{1}=\max \left\{\frac{1}{2}\left(s_{1}(1-\alpha)-s_{2}+T_{2}-\frac{s_{1}^{2}-s_{2}^{2}}{2} \underline{\Gamma}\right), 0\right\}
$$

As for school 2 , the first derivative of $\Pi_{2}$ w.r.t. $T_{2}$ is

$$
\frac{\partial \Pi_{2}}{\partial T_{2}}= \begin{cases}\frac{\left(s_{1}^{2}-s_{2}^{2}\right) \bar{\Gamma}-2\left(s_{1}-s_{2}(1-\alpha)-T_{1}+2 T_{2}\right)}{\left(s_{1}^{2}-s_{2}^{2}\right)(\bar{\Gamma}-\bar{\Gamma})}, & \text { if } T_{2}<s_{2}\left(1-\frac{s_{2}}{2} \bar{\Gamma}\right) ; \\ \frac{2\left[s_{1}^{2}\left(s_{2}(1-\alpha)-2 T_{2}\right)+s_{2}^{2}\left(T_{1}-s_{1}\right)\right]}{s_{2}^{2}\left(s_{1}^{2}-s_{2}^{2}\right)(\bar{\Gamma}-\underline{\Gamma})}, & \text { otherwise. }\end{cases}
$$

As the numerators in both parts of (B.5) are linear and decreasing in $T_{2}$, they each would have, absent the restriction of their domain, a unique root. To get the global profit maximum, note first that there is a kink in $\Pi_{2}$ at the border of the domains, with $\Pi_{2}$ being steeper slightly to the left of the border than to the right:

$$
\begin{aligned}
\lim _{T_{2} \nearrow s_{2}\left(1-\frac{s_{2}}{2} \bar{\Gamma}\right)} \frac{\partial \Pi_{2}}{\partial T_{2}} & =\frac{\left(s_{1}^{2}+s_{2}^{2}\right) \bar{\Gamma}+2 T_{1}-2 s_{1}-2 s_{2}(1+\alpha)}{\left(s_{1}^{2}-s_{2}^{2}\right)(\bar{\Gamma}-\underline{\Gamma})} \\
& =\lim _{T_{2} \searrow s_{2}\left(1-\frac{s_{2}}{2} \bar{\Gamma}\right)} \frac{\partial \Pi_{2}}{\partial T_{2}}+\frac{\left(s_{1}^{2}-s_{2}^{2}\right)\left(\frac{2(1+\alpha)}{s_{2}}-\bar{\Gamma}\right)}{\left(s_{1}^{2}-s_{2}^{2}\right)(\bar{\Gamma}-\underline{\Gamma})},
\end{aligned}
$$

where the second summand is positive at the boundary of the two domains, as the first part of (B.5) exists only if $s_{2}<\frac{2}{\bar{\Gamma}}$. 
An immediate implication of this result is that the first-order condition $\frac{\partial \Pi_{2}}{\partial T_{2}}=0$ can be satisfied in at most one of the parts in (B.5). Furthermore, it may be the case that $\lim _{T_{2} \searrow s_{2}\left(1-\frac{s_{2}}{2} \bar{\Gamma}\right)} \frac{\partial \Pi_{2}}{\partial T_{2}}<0<\lim _{T_{2} \nearrow_{s_{2}}\left(1-\frac{s_{2}}{2} \bar{\Gamma}\right)} \frac{\partial \Pi_{2}}{\partial T_{2}}$, in which case the first-order condition is satisfied in neither case, and the maximum is then at the border between both cases. Combining these cases yields the best-reply curve for school 2 :

$$
T_{2}=\left\{\begin{array}{lr}
\max \left\{\frac{1}{2}\left(T_{1}-s_{1}+s_{2}(1-\alpha)+\frac{s_{1}^{2}-s_{2}^{2}}{2} \bar{\Gamma}\right), 0\right\}, & \text { if } T_{1} \leq s_{1}+s_{2}(1+\alpha)-\frac{s_{1}^{2}+s_{2}^{2}}{2} \bar{\Gamma} \\
\max \left\{s_{2}\left(1-\frac{s_{2}}{2} \bar{\Gamma}\right), 0\right\}, & \text { if } s_{1}+s_{2}(1+\alpha)-\frac{s_{1}^{2}+s_{2}^{2}}{2} \bar{\Gamma}<T_{1} \\
\max \left\{\frac{1}{2}\left(\frac{s_{2}^{2}}{s_{1}^{2}} T_{1}+s_{2}(1-\alpha)-\frac{s_{2}^{2}}{s_{1}}\right), 0\right\}, & \quad \leq s_{1}+s_{1}^{2}\left(\frac{1+\alpha}{s_{2}}-\bar{\Gamma}\right)
\end{array}\right.
$$

In the first case of (B.6), the best-reply curve is given by the first-order condition $\frac{\partial \Pi_{2}}{\partial T_{2}}=$ 0 in the first case of (B.5), and the domain of this case is obtained by the condition $\lim _{T_{2} \nearrow s_{2}\left(1-\frac{s_{2}}{2} \bar{\Gamma}\right)} \frac{\partial \Pi_{2}}{\partial T_{2}}<0$. Similarly, the best-reply curve in the third case of (B.6) solves $\frac{\partial \Pi_{2}}{\partial T_{2}}=0$ in the second case of (B.5), and the domain of this case is obtained by the condition $\lim _{T_{2} \searrow s_{2}\left(1-\frac{s_{2}}{2} \bar{\Gamma}\right)} \frac{\partial \Pi_{2}}{\partial T_{2}}>0$. The domain of the second case of (B.6) is then simply the set of $T_{2}$ between the domains of the first and the third case, and the best-reply curve in the second case is given by the requirement that $T_{2}$ is exactly on the border between both cases of (B.5). Note that school 2's best-reply curve is continuous in $T_{1}$.

The slope of school 2's best-reply curve in a $T_{1}-T_{2}$ diagram is never larger than $\frac{1}{2}$, whereas the slope of school 1's best-reply curve is 2 . Hence, there is a unique equilibrium at the intersection of both best-reply curves. If this intersection is in the first case of (B.6), equilibrium tuition fees are

$$
\begin{aligned}
& T_{1}^{*}\left(s_{1}, s_{2}\right)=\frac{s_{1}-s_{2}}{3}\left(1+\alpha-\frac{s_{1}+s_{2}}{2}(2 \underline{\Gamma}-\bar{\Gamma})\right)-\alpha s_{1} \\
& T_{2}^{*}\left(s_{1}, s_{2}\right)=\frac{s_{1}-s_{2}}{3}\left(\frac{s_{1}+s_{2}}{2}(2 \bar{\Gamma}-\underline{\Gamma})-(1+\alpha)\right)-\alpha s_{2}
\end{aligned}
$$

provided that both of these tuition fees are nonnegative. This equilibrium satisfies the definition of the first case of (B.6) if and only if

$$
s_{1}\left(1+\alpha-\frac{s_{1}}{2}(2 \bar{\Gamma}-\underline{\Gamma})\right) \geq-s_{2}\left(2(1+\alpha)-\frac{s_{2}}{2}(\bar{\Gamma}+\underline{\Gamma})\right) .
$$

We will need the slope of the boundary of the region defined by (B.9) later in the proof of Proposition 2:

$$
\frac{d s_{2}}{d s_{1}}=-\frac{1+\alpha-s_{1}(2 \bar{\Gamma}-\underline{\Gamma})}{2(1+\alpha)-s_{2}(\bar{\Gamma}+\underline{\Gamma})}
$$

It can be shown that for $0<s_{2}<s_{1}$ this boundary has the shape of an inverted C. 
Using (B.7) and (B.8) to substitute for $T_{1}$ and $T_{2}$ in (B.2) and (B.3) then yields (13) and (14).

We briefly mention the other cases of (B.6) as we will need them in the proof of Proposition 2: If equilibrium is in the second case of (B.6), equilibrium tuition fees are

$$
\begin{aligned}
& T_{1}^{*}\left(s_{1}, s_{2}\right)=\frac{1}{2}\left(s_{1}(1+\alpha)-\frac{s_{2}^{2} \bar{\Gamma}+\left(s_{1}^{2}-s_{2}^{2}\right) \underline{\Gamma}}{2}\right)-\alpha s_{1} \\
& T_{2}^{*}\left(s_{1}, s_{2}\right)=s_{2}\left(1-\frac{s_{2}}{2} \bar{\Gamma}\right),
\end{aligned}
$$

provided that both are nonnegative, which is the case for $T_{1}^{*}$ if and only if

$$
s_{1}\left(1-\alpha-\frac{s_{1}}{2} \underline{\Gamma}\right)-\frac{s_{2}^{2}}{2}(\bar{\Gamma}-\underline{\Gamma}) \geq 0 .
$$

This equilibrium satisfies the definition of the first case of (B.6) if and only if the reverse of (B.9) is true, and at the same time

$$
\frac{s_{1}^{2}}{s_{2}}\left(1+\alpha-\frac{s_{2}}{2}(2 \bar{\Gamma}-\underline{\Gamma})\right)+s_{1}(1+\alpha)+\frac{s_{2}^{2}}{2}(\bar{\Gamma}-\underline{\Gamma}) \geq 0 .
$$

If (B.14) does not hold, school 2's equilibrium tuition fees in the third case of (B.6) apply, but we will not analyze this case in detail as we will show in the proof of Proposition 2 that it is irrelevant for our analysis.

\section{Appendix C. Proof of Proposition 2}

We start by proving the characterization of the interior equilibrium given in part (i) and the boundary equilibrium given in part (ii) of the Proposition along with the proof of the comparative statics given in part (iii). Then, we will prove that it is not profitable for any school to deviate to a curriculum outside the set which governs Lemma 1. Last, we will prove part (iv) of the Proposition.

\section{Appendix C.1. Characterization of the Interior Solution}

The partial derivatives of equilibrium profits in the pricing stage (13) and (14) with respect to the respective school's choice of curriculum are

$$
\begin{aligned}
\frac{\partial \Pi_{1}^{*}}{\partial s_{1}} & =\frac{4\left(1+\alpha-\frac{s_{1}+s_{2}}{2}(2 \underline{\Gamma}-\bar{\Gamma})\right)}{9\left(s_{1}+s_{2}\right)^{2}(\bar{\Gamma}-\underline{\Gamma})}\left(s_{2}(1+\alpha)-s_{1} \frac{s_{1}+s_{2}}{2}(2 \underline{\Gamma}-\bar{\Gamma})\right) \\
\frac{\partial \Pi_{2}^{*}}{\partial s_{2}} & =\frac{4\left(\frac{s_{1}+s_{2}}{2}(2 \bar{\Gamma}-\underline{\Gamma})-(1+\alpha)\right)}{9\left(s_{1}+s_{2}\right)^{2}(\bar{\Gamma}-\underline{\Gamma})}\left(s_{1}(1+\alpha)-s_{2} \frac{s_{1}+s_{2}}{2}(2 \bar{\Gamma}-\underline{\Gamma})\right) .
\end{aligned}
$$

Let us start with the laxer school's profit, which has two local extremal points $s_{2}^{a}$ and $s_{2}^{b}$, given implicitly by

$$
1+\alpha=\frac{s_{1}+s_{2}^{a}}{2}(2 \bar{\Gamma}-\underline{\Gamma})
$$


and

$$
1+\alpha=\frac{s_{2}^{b}}{s_{1}} \frac{s_{1}+s_{2}^{b}}{2}(2 \bar{\Gamma}-\underline{\Gamma}) .
$$

both of which always exist because $\bar{\Gamma}>\underline{\Gamma}$. Note that the right-hand side of the former condition is always larger than the right-hand side of the latter condition, and both righthand sides are monotonically increasing in $s_{2}$. Hence, $s_{2}^{a}<s_{2}^{b}$. Furthermore, this implies that in a small neighborhood below $s_{2}^{b}$, both the numerator of the fraction on the right-hand side of (C.2) and the large bracket in (C.2) are positive, proving that there is a local maximum at $s_{2}^{b}$. One can show by a similar argument that there is a local minimum at $s_{2}^{a}$. Furthermore, $s_{2}^{a}$ is the lower boundary of the laxer school's feasible set, because curricula below this would lead to negative demand and negative equilibrium 'gross price' $T_{2}+\alpha s_{2}$. Hence, the global maximum is at $s_{2}^{b}$ which satisfies the latter first-order condition (C.4).

For the tougher school's profit, interior local extremal points exist if and only if $\bar{\Gamma}<2 \underline{\Gamma}$, an assumption we made in Section 2. Then, the argument is very similar to that for the laxer school: There are two extremal points at $s_{1}^{a}$ and $s_{1}^{b}$ defined by

$$
1+\alpha=\frac{s_{1}^{a}+s_{2}}{2}(2 \underline{\Gamma}-\bar{\Gamma})
$$

and

$$
1+\alpha=\frac{s_{1}^{b}}{s_{2}} \frac{s_{1}^{b}+s_{2}}{2}(2 \underline{\Gamma}-\bar{\Gamma}),
$$

with $s_{1}^{a}>s_{1}^{b}$. Again, both the numerator and the term in large brackets on the right-hand side of (C.1) are positive for $s_{1}<s_{1}^{b}$ slightly below $s_{1}^{b}$, which proves that there is a local maximum at $s_{1}^{b}$. Furthermore, there is a local minimum at $s_{1}^{a}$, which is the upper bound of the tougher school's feasible set. Hence, the global maximum is at $s_{1}^{b}$ given by (C.6).

Substituting for $s_{1}=s_{1}^{b}$ in (C.4) and for $s_{2}=s_{2}^{b}$ in (C.6), and combining both yields

$$
\frac{s_{2}^{b}}{s_{1}^{b}}=\sqrt{\frac{\bar{\Gamma}-\frac{\bar{\Gamma}}{2}}{\bar{\Gamma}-\frac{\Gamma}{2}}}
$$

and, substituting in (C.4) and (C.6), equilibrium curriculum choices (15) and (16).

Let us now compare equilibrium curricula $s_{1}^{e}, s_{2}^{e}$ with the efficient curricula $s_{1}^{f}, s_{2}^{f}$ derived in Proposition 1: 


$$
\begin{aligned}
s_{1}^{e} & =\frac{2(1+\alpha)}{\sqrt{2 \underline{\Gamma}-\bar{\Gamma}}(\sqrt{2 \bar{\Gamma}-\underline{\Gamma}+\sqrt{2 \underline{\Gamma}-\bar{\Gamma}})}} \\
& =\frac{2(1+\alpha)}{\sqrt{2 \underline{\Gamma}-\bar{\Gamma}} \sqrt{2 \bar{\Gamma}-\underline{\Gamma}+2 \sqrt{(2 \bar{\Gamma}-\underline{\Gamma})(2 \underline{\Gamma}-\bar{\Gamma})}+2 \underline{\Gamma}-\bar{\Gamma}}} \\
& =\frac{2(1+\alpha)}{\sqrt{\underline{\Gamma}-(\bar{\Gamma}-\underline{\Gamma})} \sqrt{\bar{\Gamma}+2 \sqrt{\bar{\Gamma} \underline{\Gamma}-2(\bar{\Gamma}-\underline{\Gamma})^{2}}+\underline{\Gamma}}} \\
& >\frac{2(1+\alpha)}{\sqrt{\underline{\Gamma}(\sqrt{\bar{\Gamma}}+\sqrt{\underline{\Gamma}})}}
\end{aligned}
$$

which would be equal to $s_{1}^{f}$ for $\alpha=\beta$.

$$
\begin{aligned}
& s_{2}^{e}=\frac{2(1+\alpha)}{\sqrt{2 \bar{\Gamma}-\underline{\Gamma}}(\sqrt{2 \bar{\Gamma}-\underline{\Gamma}}+\sqrt{2 \underline{\Gamma}-\bar{\Gamma}})} \\
& =\frac{2(1+\alpha)}{\bar{\Gamma}+(\bar{\Gamma}-\underline{\Gamma})+\sqrt{\bar{\Gamma} \underline{\Gamma}-2(\bar{\Gamma}-\underline{\Gamma})^{2}}} \\
& =\frac{2(1+\alpha)}{\bar{\Gamma}+\sqrt{(\bar{\Gamma}-\underline{\Gamma})^{2}+2(\bar{\Gamma}-\underline{\Gamma}) \sqrt{\bar{\Gamma} \underline{\Gamma}-2(\bar{\Gamma}-\underline{\Gamma})^{2}}+\bar{\Gamma} \underline{\Gamma}-2(\bar{\Gamma}-\underline{\Gamma})^{2}}}
\end{aligned}
$$

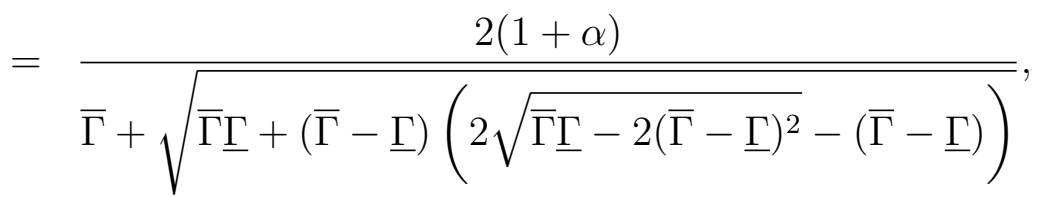

which is smaller than $\frac{2(1+\alpha)}{\bar{\Gamma}+\sqrt{\bar{\Gamma}} \underline{\Gamma}}$ if and only if $4 \bar{\Gamma} \underline{\Gamma}>9(\bar{\Gamma}-\underline{\Gamma})^{2}$, which is satisfied if and only if $\bar{\Gamma}<1.92495 \cdot \underline{\Gamma}$. It will be shown in Subsection Appendix C.2 that this is always the case if the equilibrium is the interior solution.

Appendix C.2. Characterization of the Boundary Solution

We shall now derive for which parameters the definition of the first case in (B.6) binds. Define

$$
\begin{aligned}
\bar{G} & :=\sqrt{2 \bar{\Gamma}-\underline{\Gamma}} \\
\underline{G} & :=\sqrt{2 \underline{\Gamma}-\bar{\Gamma}}
\end{aligned}
$$


so that $s_{1}^{e}=\frac{2(1+\alpha)}{\underline{G}(\bar{G}+\underline{G})}, s_{2}^{e}=\frac{2(1+\alpha)}{\bar{G}(\bar{G}+\underline{G})}, s_{1}^{e}+s_{2}^{e}=\frac{2(1+\alpha)}{\bar{G} \underline{G}}$ and $s_{1}^{e}-s_{2}^{e}=\frac{2(1+\alpha)(\bar{G}-\underline{G})}{\bar{G} \underline{G}(\bar{G}+\underline{G})}$. Then, applying (B.9) to the curricula $s_{1}^{e}$ and $s_{2}^{e}$ yields

$$
\begin{aligned}
& s_{1}^{e}(1+\alpha-\frac{s_{1}^{e}}{2} \underbrace{(2 \bar{\Gamma}-\underline{\Gamma})}_{=\bar{G}^{2}})+s_{2}^{e}(2(1+\alpha)-\frac{s_{2}^{e}}{2} \underbrace{(\bar{\Gamma}+\underline{\Gamma})}_{=\bar{G}^{2}+\underline{G}^{2}}) \geq 0 \\
& \frac{2(1+\alpha)^{2}}{\underline{G}(\bar{G}+\underline{G})}\left(1-\frac{\bar{G}^{2}}{\underline{G}(\bar{G}+\underline{G})}\right)+\frac{2(1+\alpha)^{2}}{\bar{G}(\bar{G}+\underline{G})}\left(2-\frac{\bar{G}^{2}+\underline{G}^{2}}{\bar{G}(\bar{G}+\underline{G})}\right) \geq 0 \\
& \frac{2(1+\alpha)^{2}}{\bar{G}^{2} \underline{G}^{2}(\bar{G}+\underline{G})^{2}}\left[\bar{G} \underline{G}\left(\bar{G}^{2}+2 \underline{G}^{2}\right)-\left(\bar{G}^{2}-\underline{G}^{2}\right)^{2}\right] \geq 0 \\
& \frac{6(1+\alpha)^{2}}{\bar{G}^{2} \underline{G}^{2}(\bar{G}+\underline{G})^{2}}\left[\bar{G} \underline{G \Gamma}-3(\bar{\Gamma}-\underline{\Gamma})^{2}\right] \geq 0,
\end{aligned}
$$

which is satisfied if and only if $\bar{\Gamma} \leq 1.566210 \cdot \underline{\Gamma}$.

Consider now the case where $s_{1}^{e}$ and $s_{2}^{e}$ are outside the first case of (B.6). Recall from (B.10) that the boundary between the first and the second case of (B.6) has the shape of an inverted $\mathrm{C}$ in the $s_{1}-s_{2}$ diagram with the unique maximum $s_{1}$ being at $s_{2}=\frac{2(1+\alpha)}{\bar{\Gamma}+\underline{\Gamma}}$. Furthermore,

$$
s_{2}^{e}=\frac{2(1+\alpha)}{\bar{G}(\bar{G}+\underline{G})}<\frac{2(1+\alpha)}{\bar{G}^{2}+\underline{G}^{2}}=\frac{2(1+\alpha)}{\bar{\Gamma}+\underline{\Gamma}} .
$$

Last, both schools' best-reply curves given by (C.6) and (C.4) are strictly upward sloping in the $s_{1}-s_{2}$ diagram, school 2's best-reply curve being located above school 1's for $s_{1}<s_{1}^{e}$. Hence, if $s_{1}^{e}$ and $s_{2}^{e}$ are outside the first case of (B.6), both schools' best-reply curves intersect this boundary where it is upward sloping in the $s_{1}-s_{2}$ diagram, and the candidate equilibrium in the boundary case is given by the intersection of (C.4) and the equality version of (B.9).

For comparative statics and comparisons with first-best curricula, we need a better characterization of the boundary solution. Solving (C.4) for $s_{1}$ yields $s_{1}=\frac{s_{2}^{2}(2 \bar{\Gamma}-\underline{\Gamma})}{2(1+\alpha)-s_{2}(2 \bar{\Gamma}-\underline{\Gamma})}$, which we can use to substitute for $s_{1}$ in the equality version of (B.9), which after some rearranging gives us

$$
B\left(s_{2}\right):=-\frac{3}{2} s_{2}^{3}(2 \bar{\Gamma}-\underline{\Gamma})^{2} \bar{\Gamma}+s_{2}^{2}(1+\alpha)(2 \bar{\Gamma}-\underline{\Gamma})(4 \bar{\Gamma}+\underline{\Gamma})-2 s_{2}(1+\alpha)^{2}(7 \bar{\Gamma}-2 \underline{\Gamma})+8(1+\alpha)^{3}=0 .
$$

We can get comparative statics by taking the total differential of $B\left(s_{2}\right)$ on the left-hand side of (C.10) with $\frac{d s_{2}}{d \alpha}=-\frac{\frac{\partial B}{\partial \alpha}}{\frac{\partial B}{\partial s_{2}}}$. If $B\left(s_{2}\right)=0$, then

$$
\begin{aligned}
\frac{\partial B}{\partial s_{2}} & =-\frac{9}{2} s_{2}^{2}(2 \bar{\Gamma}-\underline{\Gamma})^{2} \bar{\Gamma}+2 s_{2}(1+\alpha)(2 \bar{\Gamma}-\underline{\Gamma})(4 \bar{\Gamma}+\underline{\Gamma})-2(1+\alpha)^{2}(7 \bar{\Gamma}-2 \underline{\Gamma}) \\
& =-s_{2}(1+\alpha)(2 \bar{\Gamma}-\underline{\Gamma})(4 \bar{\Gamma}+\underline{\Gamma})+4(1+\alpha)^{2}(7 \bar{\Gamma}-2 \underline{\Gamma})-24 \frac{(1+\alpha)^{3}}{s_{2}}
\end{aligned}
$$


where the second equality uses $B\left(s_{2}\right)=0$ to substitute for $\frac{3}{2} s_{2}^{2}(2 \bar{\Gamma}-\underline{\Gamma})^{2} \bar{\Gamma}$. This rearranged expression is strictly increasing in $s_{2}$ whenever $s_{2}<\frac{2(1+\alpha)}{\bar{\Gamma}+\underline{\Gamma}}$, which we can use as an upper bound, which yields $\frac{\partial B}{\partial s_{2}}<-\frac{36(1+\alpha)^{2}}{(\bar{\Gamma}+\underline{\Gamma})^{2}} \underline{\Gamma}^{2}<0$.

Furthermore,

$$
\frac{\partial B}{\partial \alpha}=s_{2}^{2}(2 \bar{\Gamma}-\underline{\Gamma})(4 \bar{\Gamma}+\underline{\Gamma})-4 s_{2}(1+\alpha)(7 \bar{\Gamma}-2 \underline{\Gamma})+24(1+\alpha)^{2},
$$

which is decreasing in $s_{2}$ whenever $s_{2}<\frac{2(1+\alpha)}{\bar{\Gamma}+\underline{\Gamma}}$, which we can use as a lower bound, which yields $\frac{\partial B}{\partial \alpha}<-\frac{36(1+\alpha)^{2}}{(\bar{\Gamma}+\underline{\Gamma})^{2}} \underline{\Gamma}^{2}<0$. Hence, $\frac{d s_{2}}{d \alpha}>0$.

In the $s_{1}-s_{2}$ diagram, It is easy to check that an increase in $\alpha$ will move school 2's bestreply curve to the left and the boundary of the first case of (B.6) to the right. Furthermore, both curves are positively sloped, and school 2's best-reply curve is the flatter of both curves. Hence, the intersection of these curves will move to the right, which proves that $\frac{d s_{1}}{d \alpha}>0$.

Let us now compare the boundary solution to the first-best curricula. Suppose first that

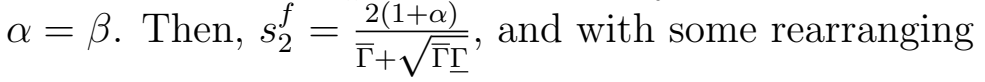

$$
B\left(s_{2}^{f}\right)=\frac{1+\alpha}{(\bar{\Gamma}+\sqrt{\bar{\Gamma} \underline{\Gamma}})^{3}}\left[\bar{\Gamma}\left(-9 \bar{\Gamma}^{2}+11 \bar{\Gamma} \underline{\Gamma}-\underline{\Gamma}^{2}\right)+\sqrt{\bar{\Gamma} \underline{\Gamma}} \underline{\Gamma}(4 \bar{\Gamma}-\underline{\Gamma})\right],
$$

which is negative whenever $\bar{\Gamma}$ is so large that the equilibrium is the boundary solution. Hence, school 2's curriculum in the boundary solution is below $s_{2}^{f}$ if $\alpha=\beta$. As $\frac{d s_{2}}{d \alpha}>0$, this holds for all $0 \leq \alpha \leq \beta$.

\section{Appendix C.3. Deviations to Other Cases}

We will now prove that for sufficiently low $\alpha$ it is not possible for a school to profitably deviate to some curriculum outside the area in which profits are given by (13) and (14). To begin with, it is important to note that the (piecewise) profit functions are continuous everywhere. Furthermore, if $s_{1}^{e}$ and $s_{2}^{e}$ are in the first case of (B.6), then the resulting equilibrium tuition fees are strictly positive for sufficiently low $\alpha$ : Using the notations introduced in (C.8) and (C.9) to substitute for $s_{1}$ and $s_{2}$ in (B.7) and (B.8), we get

$$
\begin{aligned}
& T_{1}=\frac{2(1+\alpha)}{\underline{G(\bar{G}+\underline{G})}}\left(\frac{(\bar{G}-\underline{G})^{2}(1+\alpha)}{3 \bar{G}^{2}}-\alpha\right) \\
& T_{2}=\frac{2(1+\alpha)}{\bar{G}(\bar{G}+\underline{G})}\left(\frac{(\bar{G}-\underline{G})^{2}(1+\alpha)}{3 \underline{G}^{2}}-\alpha\right),
\end{aligned}
$$

for which both right-hand sides have a strictly positive first summand even for $\alpha=0$, so that it is always possible to find some positive, but very small $\alpha$ such that tuition fees are strictly positive. Furthermore, together with continuity of the profit functions and the fact that their derivatives are always finite as long as $s_{1}>s_{2}$, this implies that it is always possible to find some positive and very small $\alpha$ such that school $k$ 's maximum profit with $T_{i}=0$ for some $i$ and for given curriculum of the rival school $s_{-k}=s_{-k}^{e}$ is smaller than its profit under curricula $s_{1}^{e}, s_{2}^{e}$. Hence, we can rule out deviations to zero tuition fees within the first case of (B.6). It remains to show that schools will not deviate to other cases in (B.6). 
School 1 deviates. Let us start with school 1. Note first that school 1 can never deviate from the candidate equilibrium to the third case in (B.6), which is defined by the complementary set of (B.14). As the equilibrium $s_{2} \leq s_{2}^{e}=\frac{2(1+\alpha)}{\bar{G}^{2}+\bar{G} G}<\frac{2(1+\alpha)}{\bar{G}^{2}}$, the left-hand side of (B.14) is positive for all $s_{1}$. Hence, for every $s_{2} \in\left(0, s_{1}\right)$, there exists a $s_{1}^{\prime}\left(s_{2}\right)$ such that $\left(s_{1}, s_{2}\right)$ is in the first case of (B.6) if and only if $s_{1} \leq s_{1}^{\prime}\left(s_{2}\right)$, and in the second case otherwise. Now, we fix school 2's curriculum at $s_{2}^{e}$. The following Lemma shows that if $\left(s_{1}^{e}, s_{2}^{e}\right)$ are in the first case of (B.6), then school 1 cannot increase profit by unilaterally deviating to the second case of (B.6).

Lemma 4. In the second case of (B.6) with positive tuition fees, school 1's profit is strictly decreasing in $s_{1}$ for every $s_{2}$.

Proof. This case is defined by the intersection of the sets of $\left(s_{1}, s_{2}\right)$ satisfying $s_{2} \geq 0$, the reverse of (B.9) and (B.13). ${ }^{14}$ School 1's profit is

$$
\Pi_{1}^{*}=\frac{\left[s_{1}\left(1+\alpha-\frac{s_{1}}{2} \underline{\Gamma}\right)-\frac{s_{2}^{2}}{2}(\bar{\Gamma}-\underline{\Gamma})\right]^{2}}{2\left(s_{1}^{2}-s_{2}^{2}\right)(\bar{\Gamma}-\underline{\Gamma})},
$$

the first derivative of which is

$$
\frac{\partial \Pi_{1}^{*}}{\partial s_{1}}=-\frac{\left[s_{1}\left(1+\alpha-\frac{s_{1}}{2} \underline{\Gamma}\right)-\frac{s_{2}^{2}}{2}(\bar{\Gamma}-\underline{\Gamma})\right]\left[s_{2}^{2}\left(1+\alpha-\frac{s_{1}}{2} \bar{\Gamma}\right)+\frac{s_{1}}{2}\left(s_{1}^{2}-s_{2}^{2}\right) \underline{\Gamma}\right]}{\left(s_{1}^{2}-s_{2}^{2}\right)^{2}(\bar{\Gamma}-\underline{\Gamma})} .
$$

The denominator is always positive, and the first term in solid brackets in the numerator is positive as long as demand is positive. In the $s_{1}-s_{2}$ diagram the roots to this first solid bracket are on an inverted-U-shaped curve which intersects the 45 -degree line at $s_{1}=s_{2}=\frac{2(1-\alpha)}{\bar{\Gamma}}$ and which is downward sloping for $s_{1}>s_{2}>0$. The second case of (B.6) with positive tuition fees is a subset of (B.13), which is defined by $s_{1}\left(1-\alpha-\frac{s_{1}}{2} \underline{\Gamma}\right)-\frac{s_{2}^{2}}{2}(\bar{\Gamma}-\underline{\Gamma}) \geq 0$, the left-hand side of which is equal to the first term in solid brackets in (C.12) less $2 \alpha s_{1}$, which proves that the first term in solid brackets in (C.12) is always positive in the second case of (B.6) with positive tuition fees.

Concerning the second term in solid brackets, $s_{1}>s_{2}$ implies that it is larger than $s_{2}^{2}\left(1+\alpha-\frac{s_{1}}{2} \bar{\Gamma}\right)$, which is positive if and only if $s_{1}<\frac{2(1+\alpha)}{\bar{\Gamma}}$. The roots to this term are located on a curve which intersects the 45-degree line in the $s_{1}-s_{2}$ diagram at $s_{1}=\frac{2(1+\alpha)}{\bar{\Gamma}}$ and is strictly increasing for $s_{1}>\frac{2(1+\alpha)}{\bar{\Gamma}}$ : This curve on which the roots of the second term in solid brackets are located is given by

$$
s_{2}^{2}=\frac{s_{1}^{3} \underline{\Gamma}}{s_{1}(\bar{\Gamma}+\underline{\Gamma})-2(1+\alpha)}
$$

\footnotetext{
${ }^{14}$ Recall that (B.14) is always satisfied for school 2's equilibrium curriculum.
} 
the derivative of which is

$$
\frac{d s_{2}}{d s_{1}}=\frac{s_{1}^{2} \underline{\Gamma}\left(s_{1}(\bar{\Gamma}+\underline{\Gamma})-3(1+\alpha)\right.}{\left(s_{1}(\bar{\Gamma}+\underline{\Gamma})-2(1+\alpha)\right) s_{2}}
$$

which is positive for every $s_{1}>\frac{2(1+\alpha)}{\bar{\Gamma}}$. Hence, the right-hand side of (C.12) is negative for all $s_{2}<\frac{2(1+\alpha)}{\bar{\Gamma}}$ and therefore throughout the second case of (B.6) with positive tuition fees.

School 2 deviates to the second case with $T_{1}>0$. Let us now turn to school 2. Assume for the moment that school 1 will choose positive tuition fees in the pricing stage. Recall from (B.10) that in the $s_{1}-s_{2}$ diagram, the boundary between the first and the second case of (B.6) has the shape of an inverted C: The curve has a maximum $s_{1}$ at $s_{2}=\frac{2(1+\alpha)}{\bar{\Gamma}+\underline{\Gamma}}$ and is downward (upward) sloping for larger (smaller) $s_{2}$.

For any given pair of curricula $s_{1}, s_{2}$ which belongs to one of the first two cases of (B.6), school 2's profit at equilibrium tuition fees can be calculated using (B.3):

$$
\Pi_{2}^{*}\left(s_{2} ; s_{1}\right)=\Pi_{2}\left(T_{2}^{*} ; T_{1}^{*}, s_{1}, s_{2}\right)=\left(T_{2}^{*}+\alpha s_{2}\right) \frac{\bar{\Gamma}-\frac{2}{s_{1}+s_{2}}\left(1-\frac{T_{1}^{*}-T_{2}^{*}}{s_{1}-s_{2}}\right)}{\bar{\Gamma}-\underline{\Gamma}}
$$

Hence, the effect of school 2's choice of curriculum on equilibrium profit can be written as

$$
\frac{\partial \Pi_{2}^{*}\left(s_{2} ; s_{1}\right)}{\partial s_{2}}=\frac{\partial \Pi_{2}\left(T_{2}^{*} ; T_{1}^{*}, s_{1}, s_{2}\right)}{\partial s_{2}}+\frac{\partial \Pi_{2}\left(T_{2}^{*} ; T_{1}^{*}, s_{1}, s_{2}\right)}{\partial T_{2}} \frac{d T_{2}^{*}}{d s_{2}}+\frac{\partial \Pi_{2}\left(T_{2}^{*} ; T_{1}^{*}, s_{1}, s_{2}\right)}{\partial T_{1}} \frac{d T_{1}^{*}}{d s_{2}} .
$$

The candidate equilibria are always located in the first case of (B.6) and clearly maximize schools' profits within this case. Outside this case, deviations to the second case of (B.6) may be possible with larger and smaller $s_{2}$, due to the inverted-C shape of the boundary between the first and the second case of (B.6) in the $s_{1}-s_{2}$ diagram. That is to say, for given $s_{1}$ the set of $s_{2}$ satisfying the first case of (B.6) may have an upper boundary and a lower boundary at the same time. Let us first compare on both sides of such a boundary. As the candidate equilibrium is always on school 2's best-reply curve, it must be the case that the limit of $\frac{\partial \Pi_{2}^{*}\left(s_{2} ; s_{1}\right)}{\partial s_{2}}$ as $s_{2}$ approaches the upper (lower) boundary of the first case is non-positive (non-negative).

To be more specific, note first that $\frac{\partial \Pi_{2}\left(T_{2}^{*} ; T_{1}^{*}, s_{1}, s_{2}\right)}{\partial T_{2}}=0$ in the first case of (B.6) with $T_{2}^{*}>0$. This is in general not true in the second case, but as $\frac{\partial \Pi_{2}\left(T_{2}^{*} ; T_{1}^{*}, s_{1}, s_{2}\right)}{\partial T_{2}}$ is continuous, it must be satisfied in the limit where $s_{2}$ is close to the boundary between the two cases. Furthermore, $\frac{\partial \Pi_{2}\left(T_{2}^{*} ; T_{1}^{*}, s_{1}, s_{2}\right)}{\partial s_{2}}$ and $\frac{\partial \Pi_{2}\left(T_{2}^{*} ; T_{1}^{*}, s_{1}, s_{2}\right)}{\partial T_{1}}$ are independent of whether we are in the first or in the second case of (B.6). As $\frac{\partial \Pi_{2}\left(T_{2}^{*} ; T_{1}^{*}, s_{1}, s_{2}\right)}{\partial T_{1}}>0$, the limit of $\frac{\partial \Pi_{2}^{*}\left(s_{2} ; s_{1}\right)}{\partial s_{2}}$ as $s_{2}$ approaches the boundary in the first case is larger than the limit as $s_{2}$ approaches the boundary in the second case if and only if $\frac{d T_{1}^{*}}{d s_{2}}$ is larger in the first case than in the second case. In the first case, $\frac{d T_{1}^{*}}{d s_{2}}=-\frac{1}{3}\left(1+\alpha-s_{2}(2 \underline{\Gamma}-\bar{\Gamma})\right)$, and in the second case it is $\frac{d T_{1}^{*}}{d s_{2}}=-\frac{1}{2} s_{2}(\bar{\Gamma}-\underline{\Gamma})$. The former is larger than the latter if and only if $s_{2}>\frac{2(1+\alpha)}{\bar{\Gamma}+\underline{\Gamma}}$, which is exactly where the 
maximum $s_{1}$ on the boundary between the first and the second case is. Hence, $\frac{\partial \Pi_{2}^{*}\left(s_{2} ; s_{1}\right)}{\partial s_{2}}$ is always larger if $s_{2}$ approaches the boundary from below than if it approaches it from above. This proves that a deviation to the second case close to the boundary is never profitable.

To complete the proof that a deviation to any $s_{2}$ in the second case is unattractive, we only have to rule out a local minimum in the second case. To this end, let us substitute for $T_{1}^{*}$ and $T_{2}^{*}$ in school 2's optimal profit in the second case, which yields

$$
\Pi_{2}^{*}\left(s_{2} ; s_{1}\right)=s_{2}\left(1+\alpha-\frac{s_{2}}{2} \bar{\Gamma}\right)\left(\frac{1}{2}+\frac{s_{1}\left(\frac{s_{1}}{2} \bar{\Gamma}-(1+\alpha)\right)}{(\bar{\Gamma}-\underline{\Gamma})\left(s_{1}^{2}-s_{2}^{2}\right)}\right)
$$

and

$$
\frac{\partial \Pi_{2}^{*}\left(s_{2} ; s_{1}\right)}{\partial s_{2}}=\frac{1+\alpha-s_{2} \bar{\Gamma}}{2}+\frac{s_{1}\left(\frac{s_{1}}{2} \bar{\Gamma}-(1+\alpha)\right)}{(\bar{\Gamma}-\underline{\Gamma})\left(s_{1}^{2}-s_{2}^{2}\right)^{2}}\left[s_{1}^{2}\left(1+\alpha-s_{2} \bar{\Gamma}\right)+s_{2}^{2}(1+\alpha)\right] .
$$

If $s_{2}=0$, then $\frac{\partial \Pi_{2}^{*}\left(s_{2} ; s_{1}\right)}{\partial s_{2}}=\frac{1+\alpha}{2}\left(1+\frac{\bar{\Gamma}-\frac{2(1+\alpha)}{s_{1}}}{\bar{\Gamma}-\underline{\Gamma}}\right)$, which is positive if and only if $s_{1}>\frac{2(1+\alpha)}{2 \bar{\Gamma}-\underline{\Gamma}}$, which is in turn the minimum $s_{1}$ on the boundary between the first and the second case for $s_{1}>s_{2} \geq 0$. Furthermore, the first summand on the right-hand side of (C.15) is strictly decreasing and the second summand strictly increasing in $s_{2}$. Hence, there cannot be any root to $\frac{\partial \Pi_{2}^{*}\left(s_{2} ; s_{1}\right)}{\partial s_{2}}$ between $s_{2}=0$ and the lower boundary of the first case of (B.6).

On the other hand, if $s_{2}$ approaches $s_{1}$, the denominator in the second summand on the right-hand side of (C.15) approaches zero. Furthermore, the expression in solid brackets is smaller than $s_{1}^{2}\left(2(1+\alpha)-s_{2} \bar{\Gamma}\right)$, which is negative for $s_{2}$ close to $s_{1}$ whenever $s_{1}>\frac{2(1+\alpha)}{\bar{\Gamma}}$, which is the intersection of the boundary with the main diagonal in the $s_{1}-s_{2}$ diagram. Hence, as the other factors in this summand are all positive when $s_{2}$ is slightly below $s_{1}$ and $s_{1}>\frac{2(1+\alpha)}{2 \bar{\Gamma}-\underline{\Gamma}}$, this second summand is infinitely negative. As the first summand is finite, it follows that $\frac{\partial \Pi_{2}^{*}\left(s_{2} ; s_{1}\right)}{\partial s_{2}}$ is negative in the second case of (B.6) as $s_{2}$ approaches $s_{1}$. As both summands are again monotonic, it also follows that this derivative is negative for all $s_{2}$ between the boundary between the cases and the main diagonal.

School 2 deviates to the second case with $T_{1}=0$. For the case of $T_{1}=0$, school 2's profit and its derivative are

$$
\begin{aligned}
\Pi_{2}^{*}\left(s_{2} ; s_{1}\right) & =\frac{s_{1}\left(1+\alpha-\frac{s_{2}}{2} \bar{\Gamma}\right) s_{1}\left(\bar{\Gamma} s_{1}-2\right)}{\left(s_{1}^{2}-s_{2}^{2}\right)(\bar{\Gamma}-\underline{\Gamma})} \\
\frac{\partial \Pi_{2}^{*}\left(s_{2} ; s_{1}\right)}{\partial s_{2}} & =\frac{s_{2}^{2}(1+\alpha)-s_{1}^{2}\left(s_{2} \bar{\Gamma}-(1+\alpha)\right)}{\left(s_{1}^{2}-s_{2}^{2}\right)^{2}(\bar{\Gamma}-\underline{\Gamma})} s_{1}\left(\bar{\Gamma} s_{1}-2\right) .
\end{aligned}
$$

Hence, school 2's best-reply curve is given by

$$
s_{1}^{2}\left(s_{2} \bar{\Gamma}-(1+\alpha)\right)=s_{2}^{2}(1+\alpha) .
$$

In the $s_{1}-s_{2}$ diagram, this curve intersects the main diagonal at $s_{1}=s_{2}=\frac{2(1+\alpha)}{\bar{\Gamma}}$ with infinitely negative slope, and is convex and negatively sloped for $s_{1}>\frac{2(1+\alpha)}{\bar{\Gamma}}$. Suppose now 
$\alpha=0$. Then, school 2's best-reply curve, the boundary between the first and the second cases of (B.6) and the curve above which $T_{1} \geq 0$ is binding all intersect at $s_{1}=s_{2}=\frac{2}{\bar{\Gamma}}$ and are negatively sloped. Furthermore, if $\alpha=0$ then for all $s_{1}>s_{2}$ the boundary between the first and the second cases of (B.6) is below the curve above which $T_{1} \geq 0$ is binding. As the slope of school 2's best-reply curve is infinitely negative at $s_{1}=s_{2}$, it is the lowest of the three curves for curricula close to the intersection of the three curves. Hence, it is sufficient to show that at $s_{2}=\frac{2}{\bar{\Gamma}+\underline{\Gamma}}$ the pair of curricula on school 2's best-reply curve induces school 1 to charge positive tuition fees, i.e. is still located to the left of the curve above which $T_{1} \geq 0$ is binding. Note furthermore that it is sufficient to show this for $\bar{\Gamma} \geq \frac{3}{2} \underline{\Gamma}$, as otherwise $s_{1}^{e}<\frac{2}{\bar{\Gamma}}$, so that there is no curriculum in the second case of (B.6) with $T_{1}=0$ which school 2 could possibly deviate to.

Applying $s_{2}=\frac{2}{\bar{\Gamma}+\underline{\Gamma}}$ to (C.16) under the assumption $\alpha=0$ yields

$$
s_{1}=\frac{2(1+\alpha)}{\sqrt{(\bar{\Gamma}-\underline{\Gamma})(\bar{\Gamma}+\underline{\Gamma})}}
$$

so that the left-hand side of (B.13) is

$$
\frac{2 \bar{\Gamma}^{2}-3 \bar{\Gamma} \underline{\Gamma}+3 \underline{\Gamma}^{2}-2(\bar{\Gamma}+\underline{\Gamma}) \sqrt{(\bar{\Gamma}-\underline{\Gamma})(\bar{\Gamma}+\underline{\Gamma})}}{(\bar{\Gamma}+\underline{\Gamma})^{2}(\bar{\Gamma}-\underline{\Gamma})},
$$

which is negative (at least) for all $\frac{3}{2} \underline{\Gamma} \leq \bar{\Gamma} \leq 2 \underline{\Gamma}$. Hence, we have shown that this region is unattractive for school 2 for $\alpha=0$, and because of continuity we can conclude that this holds also for positive, but sufficiently small $\alpha$.

\section{Appendix C.4. Proof of Part (iv)}

Using (B.7) and (B.8) to substitute for $T_{1}$ and $T_{2}$ in (12) yields the type of student that is indifferent between both schools for any given pair of curricula

$$
\bar{\gamma}_{1}=\frac{2(1+\alpha)}{3\left(s_{1}+s_{2}\right)}+\frac{\bar{\Gamma}+\underline{\Gamma}}{3},
$$

which is larger than the socially optimal allocation of students to schools for given curricula $s_{1}$ and $s_{2}, \frac{2(1+\beta)}{s_{1}+s_{2}}$, if and only if

$$
\frac{2+3 \beta-\alpha}{s_{1}+s_{2}}<\frac{\bar{\Gamma}+\underline{\Gamma}}{2} .
$$

For $s_{1}=s_{1}^{e}$ and $s_{2}=s_{2}^{e}$, this condition is

$$
\left(1+\frac{3(\beta-\alpha)}{2(1+\alpha)}\right) \sqrt{(2 \bar{\Gamma}-\underline{\Gamma})(2 \underline{\Gamma}-\bar{\Gamma})}<\frac{\bar{\Gamma}+\underline{\Gamma}}{2},
$$

and for $s_{1}=s_{1}^{f}$ and $s_{2}=s_{2}^{f}$, it is

$$
\left(1+\frac{\beta-\alpha}{2(1+\beta)}\right) \sqrt{\bar{\Gamma} \underline{\Gamma}}<\frac{\bar{\Gamma}+\underline{\Gamma}}{2},
$$

both of which are satisfied for sufficiently small $\beta-\alpha$. 


\section{Appendix D. Proof of Lemma 2}

We prove that for any pair of curricula $s_{1}>s_{2}$ :

- if all students have an incentive to enrol, then the less challenging school can increase profit by making its curriculum tougher (Step 1),

- and if at least the highest-cost student chooses not to enrol, then the more challenging school can increase profit by making its curriculum softer (Step 2),

and conclude that such a pair of different curricula cannot be an equilibrium.

Step 1: Suppose that all students choose to enrol, which is the case if $s_{2}-\bar{\Gamma} \frac{s_{2}^{2}}{2}-T \geq 0$. As the maximum net utility of the highest-cost student is $\frac{1}{2 \bar{\Gamma}}$, a necessary condition for full market coverage is $T \leq \frac{1}{2 \bar{\Gamma}}$. On the other hand, if this condition is satisfied, then it is always possible to find some $s_{2} \geq \frac{1}{\bar{\Gamma}}$ such that full market coverage is warranted.

Given that all students enrol, the less challenging school attracts all students between $\bar{\gamma}_{1}=\frac{2}{s_{1}+s_{2}}$ and $\bar{\Gamma}$, provided that this interval is nonempty. Hence, its profit is

$$
\Pi_{2}=\max \left\{\frac{T+\alpha s_{2}}{\bar{\Gamma}-\underline{\Gamma}}\left(\bar{\Gamma}-\frac{2}{s_{1}+s_{2}}\right), 0\right\} .
$$

If $s_{2} \leq \frac{2}{\bar{\Gamma}}-s_{1}, \Pi_{2}$ is identical to zero. For $s_{2}>\frac{2}{\bar{\Gamma}}-s_{1}$, which exists in the case considered in this step whenever $s_{1}>\frac{1}{\bar{\Gamma}}$, the derivative of $\Pi_{2}$ w.r.t. $s_{2}$ is

$$
\frac{\partial \Pi_{2}}{\partial s_{2}}=\frac{\alpha\left(\bar{\Gamma}-\frac{2}{s_{1}+s_{2}}\right)+\left(T+\alpha s_{2}\right) \frac{2}{\left(s_{1}+s_{2}\right)^{2}}}{\bar{\Gamma}-\underline{\Gamma}}>0 .
$$

Hence, if $s_{1}>\frac{1}{\bar{\Gamma}}$, the lax school can increase its profit by moving closer to the tough school's curriculum.

Finally, if $s_{1} \leq \frac{1}{\Gamma}, s_{1}$ is preferred to every $s_{2}<s_{1}$ by every student. Hence, the lax school earns zero profit in such a situation, whereas switching to a curriculum slightly tougher than $s_{1}$ would yield the (formerly) laxer school the whole market and thus strictly positive profit.

Step 2: Suppose now that the highest-cost student $\bar{\Gamma}$ chooses not to enrol, which is the case if and only if $s_{2}-\bar{\Gamma} \frac{s_{2}^{2}}{2}-T<0$. As we have restricted attention to non-negative tuition fees, the case we are dealing with in this step is characterized by

$$
T>\max \left\{s_{2}\left(1-\frac{\bar{\Gamma} s_{2}}{2}\right), 0\right\}
$$

The more challenging school attracts all students who prefer its curriculum (i) to the lax school's curriculum $\left(\gamma<\frac{2}{s_{1}+s_{2}}\right)$ and (ii) to not being enrolled $\left(\gamma<\frac{2}{s_{1}}\left(1-\frac{T}{s_{1}}\right)\right)$. Its profit is therefore

$$
\Pi_{1}=\frac{\left(T+\alpha s_{1}\right)}{\bar{\Gamma}-\underline{\Gamma}}\left(\min \left\{\frac{2}{s_{1}+s_{2}}, \frac{2}{s_{1}}\left(1-\frac{T}{s_{1}}\right)\right\}-\underline{\Gamma}\right) .
$$


Case (a). If $\frac{2}{s_{1}+s_{2}}<\frac{2}{s_{1}}\left(1-\frac{T}{s_{1}}\right)$, then the first derivative w.r.t. $s_{1}$ is

$$
\frac{\partial \Pi_{1}}{\partial s_{1}}=\frac{1}{\bar{\Gamma}-\underline{\Gamma}}\left(2 \frac{\alpha s_{2}-T}{\left(s_{1}+s_{2}\right)^{2}}-\alpha \underline{\Gamma}\right) .
$$

Suppose first that $s_{2} \leq \frac{2}{\bar{\Gamma}}$. Then, (D.1) becomes $T>s_{2}\left(1-\frac{\bar{\Gamma} s_{2}}{2}\right)$, so that

$$
\begin{aligned}
\frac{\partial \Pi_{1}}{\partial s_{1}} & <\frac{1}{\bar{\Gamma}-\underline{\Gamma}}\left(2 s_{2} \frac{\frac{\bar{\Gamma} s_{2}}{2}-(1-\alpha)}{\left(s_{1}+s_{2}\right)^{2}}-\alpha \underline{\Gamma}\right) \\
& <\frac{1}{\bar{\Gamma}-\underline{\Gamma}}\left(\frac{\bar{\Gamma}}{4}-\alpha \underline{\Gamma}-\frac{1-\alpha}{2 s_{2}}\right) \\
& \leq \overline{\bar{\Gamma}-\underline{\Gamma}}\left(\frac{\bar{\Gamma}}{4}-\underline{\Gamma}\right) \\
& <0
\end{aligned}
$$

where the first line uses the lower bound for $T$, the second line the definition $s_{1}>s_{2}$, the third line the supposition that $s_{2} \leq \frac{2}{\bar{\Gamma}}$, and the forth line $\bar{\Gamma}<2 \underline{\Gamma}$.

Suppose now that $s_{2}>\frac{2}{\bar{\Gamma}}$. Then, (D.1) becomes $T>0$, so that

$$
\begin{aligned}
\frac{\partial \Pi_{1}}{\partial s_{1}} & <\frac{\alpha}{\bar{\Gamma}-\underline{\Gamma}}\left(\frac{2 s_{2}}{\left(s_{1}+s_{2}\right)^{2}}-\underline{\Gamma}\right) \\
& <\frac{\alpha}{\bar{\Gamma}-\underline{\Gamma}}\left(\frac{1}{2 s_{2}^{2}}-\underline{\Gamma}\right) \\
& <\frac{\alpha}{\bar{\Gamma}-\underline{\Gamma}}\left(\frac{\bar{\Gamma}}{4}-\underline{\Gamma}\right) \\
& <0 .
\end{aligned}
$$

Case (b). If $\frac{2}{s_{1}+s_{2}} \geq \frac{2}{s_{1}}\left(1-\frac{T}{s_{1}}\right)$, then the first derivative w.r.t. $s_{1}$ is

$$
\begin{aligned}
\frac{\partial \Pi_{1}}{\partial s_{1}} & =\frac{2}{\bar{\Gamma}-\underline{\Gamma}}\left(-\frac{T}{s_{1}^{2}}\left(1-\alpha-\frac{2 T}{s_{1}}\right)-\alpha \underline{\Gamma}\right) \\
& <\frac{2 \alpha}{\bar{\Gamma}-\underline{\Gamma}}\left(\frac{T}{s_{1}^{2}}-\underline{\Gamma}\right) \\
& <\frac{2 \alpha}{\bar{\Gamma}-\underline{\Gamma}}\left(\frac{1}{2 s_{1}}-\underline{\Gamma}\right) \\
& <\frac{2 \alpha}{\bar{\Gamma}-\underline{\Gamma}}\left(\frac{\bar{\Gamma}}{2}-\underline{\Gamma}\right) \\
& <0,
\end{aligned}
$$

where the second and the third line use the fact that the definition of this case is equivalent to $\frac{T}{s_{1}} \leq \frac{s_{2}}{s_{1}+s_{2}}$, which in turn is smaller than $1 / 2$ due to $s_{1}>s_{2}$, and the forth line uses $s_{1} \geq \frac{1}{\bar{\Gamma}}$, which must be the case in order to avoid the other school to locate itself slightly above $s_{1}$ and attract the entire market. 


\section{Appendix E. Proof of Proposition 3}

The equilibrium curriculum $s^{*}$ specified in the proposition is the unique curriculum satisfying the requirement that it is the most preferred one by the median among all enrolled students. Hence, a situation with both schools choosing some curriculum $s \neq s^{*}$ cannot be an equilibrium: If $s<s^{*}$, it would be profitable to deviate to a curriculum slightly above $s$, as this would give the deviating school the same research spillover as before, but more than half of the total demand. The same argument holds for $s>s^{*}$. Furthermore, recall from Lemma 2 that there cannot be any heterogenous equilibria. Hence, we have proven that if there is a pure strategy equilibrium, it must be that specified in the Proposition.

To prove that this $s^{*}$ is indeed an equilibrium, it is sufficient to show that no deviation can be more profitable than the marginal deviation, i.e. than choosing a curriculum slightly above or below $s^{*}$. Hence, the proof will be organized in the following way: In the first step, we show that the best deviation to above is the marginal one. In the second step, we show that the the best deviation to below is the marginal one under certain conditions (steps 2a and $2 \mathrm{~b}$ ), and that these conditions are satisfied in our candidate equilibrium (step 2c).

Step 1: Deviation to above. If a school deviates to some $s_{1}>s^{*}$ and her rival sticks to $s_{2}=s^{*}$, the deviating school's profit is ${ }^{15}$

$$
\Pi_{1}=\frac{1}{\bar{\Gamma}-\underline{\Gamma}}\left(\frac{2}{s_{1}+s_{2}}-\underline{\Gamma}\right)\left(T+\alpha s_{1}\right)
$$

the derivative of which w.r.t. $s_{1}$ is

$$
\frac{\partial \Pi_{1}}{\partial s_{1}}=\frac{1}{\bar{\Gamma}-\underline{\Gamma}}\left(2 \frac{\alpha s_{2}-T}{\left(s_{1}+s_{2}\right)^{2}}-\alpha \underline{\Gamma}\right)
$$

which is positive only if

$$
\begin{aligned}
s_{1} & <\sqrt{2 \frac{\alpha s_{2}-T}{\alpha \underline{\Gamma}}}-s_{2} \\
& \leq s_{2}+2 s_{2}\left(\sqrt{\left.\frac{1}{2 s_{2} \underline{\Gamma}}-1\right)}\right. \\
& \leq s_{2}+2 s_{2}\left(\sqrt{\left.\frac{\bar{\Gamma}+\underline{\Gamma}}{4 \underline{\Gamma}}-1\right)}\right. \\
& <s_{2}+2 s_{2}\left(\sqrt{\left.\frac{3}{4}-1\right)}\right. \\
& <s_{2},
\end{aligned}
$$

\footnotetext{
${ }^{15}$ Note that all types below $\bar{\gamma}_{1}$ indeed prefer to be enrolled, as $\bar{\gamma}_{2}=\frac{2}{s_{2}}\left(1-\frac{T}{s_{2}}\right) \geq \frac{1}{s_{2}}>\frac{2}{s_{1}+s_{2}}=\bar{\gamma}_{1}$, as $T \leq \frac{\Gamma}{2} s_{2}^{2}$ and $s_{2}<\frac{1}{\underline{\Gamma}}$.
} 
a contradiction to $s_{1}$ being a deviation to above. Note that we used $T \geq 0$ for the second, $s_{2}=s^{*} \geq \frac{2}{\bar{\Gamma}+\underline{\Gamma}}$ for the third, and $\bar{\Gamma}<2 \underline{\Gamma}$ for the forth inequality.

Step 2a: Deviation to below; full market coverage. If a school deviates to below, i.e., chooses some $s_{2}<s^{*}$ while the rival school sticks to $s_{1}=s^{*}$, the case of full market coverage is characterized by

$$
\frac{2}{s_{2}}\left(1-\frac{T}{s_{2}}\right)>\bar{\Gamma}
$$

The deviating school's demand is

$$
D_{2}=\frac{\bar{\Gamma}-\bar{\gamma}_{1}}{\bar{\Gamma}-\underline{\Gamma}}=\frac{1}{\bar{\Gamma}-\underline{\Gamma}}\left(\bar{\Gamma}-\frac{2}{s_{1}+s_{2}}+\frac{2\left(T_{1}-T_{2}\right)}{s_{1}^{2}-s_{2}^{2}}\right)
$$

and, therefore,

$$
\frac{\partial D_{2}}{\partial s_{2}}=\frac{2}{(\bar{\Gamma}-\underline{\Gamma})\left(s_{1}+s_{2}\right)^{2}}>0 .
$$

With $\Pi_{2}=\left(T+\alpha s_{2}\right) D_{2}$, this implies

$$
\frac{\partial \Pi_{2}}{\partial s_{2}}=\alpha D_{2}+\left(T+\alpha s_{2}\right) \frac{\partial D_{2}}{\partial s_{2}}>0 .
$$

Step 2b: Deviation to below; incomplete market coverage. Under incomplete market coverage, the deviating (i.e., lax) school's profit is

$$
\begin{aligned}
\Pi_{2} & =\left(T+\alpha s_{2}\right) \frac{\bar{\gamma}_{2}-\bar{\gamma}_{1}}{\bar{\Gamma}-\underline{\Gamma}} \\
& =\frac{2}{\bar{\Gamma}-\underline{\Gamma}}\left(T+\alpha s_{2}\right)\left[\frac{1}{s_{2}}\left(1-\frac{T}{s_{2}}\right)-\frac{1}{s_{1}+s_{2}}\right] .
\end{aligned}
$$

Taking the derivative w.r.t. $s_{2}$ yields

$$
\begin{aligned}
\frac{\partial \Pi_{2}}{\partial s_{2}} & =\frac{2}{\bar{\Gamma}-\underline{\Gamma}}\left[\alpha\left(\frac{1}{s_{2}}\left(1-\frac{T}{s_{2}}\right)-\frac{1}{s_{1}+s_{2}}\right)-\left(T+\alpha s_{2}\right)\left(\frac{1}{s_{2}^{2}}\left(1-\frac{2 T}{s_{2}}\right)-\frac{1}{\left(s_{1}+s_{2}\right)^{2}}\right)\right] \\
& =\frac{2}{\bar{\Gamma}-\underline{\Gamma}}\left[\alpha\left(\frac{T}{s_{2}^{2}}-\frac{s_{1}}{\left(s_{1}+s_{2}\right)^{2}}\right)-\frac{T}{s_{2}^{2}}\left(\frac{s_{1}\left(s_{1}+2 s_{2}\right)}{\left(s_{1}+s_{2}\right)^{2}}-\frac{2 T}{s_{2}}\right)\right]
\end{aligned}
$$

As we will argue in the next substep, it is sufficient to prove the following Lemma:

Lemma 5. For every $T, s_{1}$ such that

$$
T \geq \min \left\{s_{1}\left(1-\frac{\bar{\Gamma}}{2} s_{1}\right), \frac{s_{1}}{4}\left(\frac{3}{4}-\alpha+\sqrt{\frac{9}{16}+\frac{\alpha}{2}+\alpha^{2}}\right)\right\},
$$

$\frac{\partial \Pi_{2}}{\partial s_{2}} \geq 0$ for all $s_{2}<s_{1}$ satisfying $\frac{2}{s_{2}}\left(1-\frac{T}{s_{2}}\right)<\bar{\Gamma}$. 
Proof. The condition $T \geq \frac{s_{1}}{4}\left(\frac{3}{4}-\alpha+\sqrt{\frac{9}{16}+\frac{\alpha}{2}+\alpha^{2}}\right)$ is constructed such that it implies that $\frac{\partial \Pi_{2}}{\partial s_{2}} \geq 0$ if $s_{2}$ is sufficiently close to $s_{1}$. This can be seen by substituting for $s_{2}=s_{1}$ and solving for $T$.

Hence, it remains to show that $\frac{\partial \Pi_{2}}{\partial s_{2}} \geq 0$ for all $s_{2}<s_{1}$. We will do so by proving that $\frac{\partial^{2} \Pi_{2}}{\partial s_{2}^{2}}>0$ whenever $\frac{\partial \Pi_{2}}{\partial s_{2}} \geq 0$ :

$$
\begin{aligned}
\frac{\partial^{2} \Pi_{2}}{\partial s_{2}^{2}} & =\frac{2}{\bar{\Gamma}-\underline{\Gamma}}\left(\frac{2 T}{s_{2}^{2}}\left(\frac{1-\alpha}{s_{2}}-\frac{s_{2}}{\left(s_{1}+s_{2}\right)^{3}}-\frac{3 T}{s_{2}^{2}}\right)+\frac{2 \alpha s_{1}}{\left(s_{1}+s_{2}\right)^{3}}\right) \\
& \leq \frac{4 T}{(\bar{\Gamma}-\underline{\Gamma}) s_{2}^{2}}\left((1-\alpha)\left(\frac{1}{s_{2}}-\frac{1}{s_{1}+s_{2}}\right)-\frac{s_{2}\left(1-s_{2}\right)}{\left(s_{1}+s_{2}\right)^{3}}+\frac{2 T}{s_{2}}\left(\frac{1}{s_{1}+s_{2}}-\frac{3}{s_{2}}\right)\right) \\
& =\frac{4 T}{(\bar{\Gamma}-\underline{\Gamma}) s_{2}^{2}}\left(\frac{(1-\alpha) s_{1}}{s_{2}\left(s_{1}+s_{2}\right)}-\frac{s_{2}\left(1-s_{2}\right)}{\left(s_{1}+s_{2}\right)^{3}}-\frac{2 T}{s_{2}} \frac{3 s_{1}+2 s_{2}}{s_{2}\left(s_{1}+s_{2}\right)}\right) \\
& <\frac{4 T}{(\bar{\Gamma}-\underline{\Gamma}) s_{2}^{2}}(\frac{s_{1}}{s_{2}\left(s_{1}+s_{2}\right)} \underbrace{\left(1-\alpha-\frac{3 s_{1}+2 s_{2}}{s_{2}}\left(\frac{3}{4}-\alpha\right)\right.}_{=-\frac{1}{2}+\alpha-3 \frac{s_{1}}{s_{2}}\left(\frac{3}{4}-\alpha\right)}-\frac{s_{2}\left(1-s_{2}\right)}{\left(s_{1}+s_{2}\right)^{3}}) \\
& <0 .
\end{aligned}
$$

For the first inequality we used the condition that $\frac{\partial \Pi_{2}}{\partial s_{2}} \geq 0$ to substitute for $\frac{2 \alpha s_{1}}{\left(s_{1}+s_{2}\right)^{3}}<$ $\frac{2 T}{s_{2}^{2}}\left(\frac{2 T}{s_{2}\left(s_{1}+s_{2}\right)}+\frac{s_{2}^{2}}{\left(s_{1}+s_{2}\right)^{3}}-\frac{1-\alpha}{s_{1}+s_{2}}\right)$, and for the second inequality we used the fact that $T \geq$ $\frac{s_{1}}{4}\left(\frac{3}{4}-\alpha+\sqrt{\frac{9}{16}+\frac{\alpha}{2}+\alpha^{2}}\right)>\frac{s_{1}}{2}\left(\frac{3}{4}-\alpha\right)$ for all $\alpha>0$.

Step 2c: Deviation to below; summary. A more detailed way of writing the $s^{*}$ given in (17) in the Proposition is

$$
s^{*}= \begin{cases}\frac{2}{\bar{\Gamma}+\underline{\Gamma}}, & \text { if } T \leq \frac{2 \underline{\Gamma}}{(\bar{\Gamma}+\underline{\Gamma})^{2}} \\ \sqrt{\frac{2 T}{\underline{\Gamma}}}, & \text { otherwise. }\end{cases}
$$

Note that $s_{2}=s^{*}$ satisfies the condition (E.3) of full market coverage if and only if $T \leq \frac{2 \underline{\Gamma}}{(\bar{\Gamma}+\underline{\Gamma})^{2}}$. Hence, it remains to show that the pairs of $\left(T, s^{*}\right)$ with $T>\frac{2 \underline{\Gamma}}{(\bar{\Gamma}+\underline{\Gamma})^{2}}$ satisfy the condition of Lemma $5, T \geq \frac{s^{*}}{4}\left(\frac{3}{4}-\alpha+\sqrt{\frac{9}{16}+\frac{\alpha}{2}+\alpha^{2}}\right)$. Using $s^{*} \geq \frac{2}{\bar{\Gamma}+\underline{\Gamma}}$, we have $T=\frac{\Gamma}{2} s^{* 2} \geq \frac{\Gamma}{\bar{\Gamma}+\underline{\Gamma}} s^{*}>$ $\frac{s^{*}}{2}>\frac{s^{*}}{4}\left(\frac{3}{4}-\alpha+\sqrt{\frac{9}{16}+\frac{\alpha}{2}+\alpha^{2}}\right)$ for all $\alpha>0$.

\section{Appendix F. Proof of Lemma 3}

Note first that the lower restriction $\bar{\gamma} \geq \underline{\Gamma}$ cannot bind, as yields zero value of the objective function and it is easy to find pairs of $s$ and $\bar{\gamma} \in[\underline{\Gamma}, \bar{\Gamma}]$ for which the objective function is strictly positive. Hence, the only relevant constraint is $\bar{\gamma} \leq \bar{\Gamma}$. 
The Kuhn-Tucker conditions of the regulator's problem are (19),

$$
\begin{aligned}
\frac{s\left(1+\beta-\frac{s}{2} \bar{\gamma}\right)}{\bar{\Gamma}-\underline{\Gamma}}-\lambda & =0 \\
\lambda(\bar{\gamma}-\bar{\Gamma}) & =0 \\
\lambda & \geq 0
\end{aligned}
$$

Using (19) to substitute for $s$ in (F.1) yields

$$
\lambda=\frac{2(1+\beta)^{2} \underline{\Gamma}}{(\bar{\Gamma}-\underline{\Gamma})(\bar{\gamma}+\underline{\Gamma})^{2}}>0
$$

which proves that the constraint $\bar{\gamma} \leq \bar{\Gamma}$ is binding.

\section{Appendix G. Proof of Proposition 4}

Recall that the regulator's problem of choosing $T$ is equivalent to choosing a pair $\left(s^{*}, \bar{\gamma}\right)$ such that $\bar{\gamma}=\frac{2}{s^{*}}-\underline{\Gamma}$. Using this to substitute for $\bar{\gamma}$ in (18) yields the regulator's objective function

$$
\frac{(1+2 \beta)\left(1-s^{*} \underline{\Gamma}\right)}{\bar{\Gamma}-\underline{\Gamma}}
$$

which is strictly decreasing in $s^{*}$. As the range of $s^{*}$ is $\left[\frac{2}{\bar{\Gamma}+\underline{\Gamma}}, \frac{1}{\Gamma}\right]$, the claim follows immediately.

\section{References}

Andersson, R., Quigley, J., Wilhelmsson, M., 2009. Urbanization, Productivity, and Innovation: Evidence from Investment in Higher Education. Journal of Urban Economics 66, $2-15$.

Benabou, R., 1993. Workings of a City: Location, Education, and Production. Quarterly Journal of Economics 108, 619-652.

Buddin, R., Zamarro, G., 2009. Teacher Qualifications and Student Achievement in Urban Elementary Schools. Journal of Urban Economics 66, 103-115.

Caucutt, E., 2002. Educational Vouchers When There Are Peer Group Effects - Size Matters. International Economic Review 43, 195-222.

De Fraja, G., Iossa, E., 2002. Competition Among Universities and the Emergence of the Elite Institution. Bulletin of Economic Research 54, 275-293.

Del Rey, E., 2001. Teaching versus Research: A Model of State University Competition. Journal of Urban Economics 49, 356-373. 
Eisenkopf, G., 2010. Peer Effects, Motivation, and Learning. Economics of Education Review 29, 364-374.

Epple, D., Newlon, E., Romano, R., 2002. Ability Tracking, School Competition, and the Distribution of Educational Benefits. Journal of Public Economics 83, 1-48.

Epple, D., Romano, R., 1998. Competition Between Private and Public Schools, Vouchers, and Peer-Group Effects. American Economic Review 88, 33-62.

Epple, D., Romano, R., 2008. Educational Vouchers and Cream Skimming. International Economic Review 49, 1395-1435.

Epple, D., Romano, R., Sieg, H., 2003. Peer effects, financial aid and selection of students into colleges and universities: an empirical analysis. Journal of Applied Econometrics 18, $501-525$.

Epple, D., Romano, R., Sieg, H., 2006. Admission, Tuition, and Financial Aid Policies in the Market for Higher Education. Econometrica 74, 885-928.

Friedman, M., 1997. Public Schools: Make Them Private. Education Economics 5, 341-344.

Gallego, F., 2006. Voucher-School Competition, Incentives and Outcomes: Evidence from Chile. Massachusetts Institute of Technology.

Gary-Bobo, R., Trannoy, A., 2008. Efficient Tuition Fees and Examinations. Journal of the European Economic Association 6, 1211-1242.

Gibbons, S., Machin, S., Silva, O., 2008. Choice, Competition, and Pupil Achievement. Journal of the European Economic Association 6, 912-947.

Hotelling, H., 1929. Stability in Competition. Economic Journal 39, 41-57.

Hoxby, C., 1999. The Productivity of Schools and Other Local Public Goods Producers. Journal of Public Economics 74, 1-30.

Hoxby, C., 2000. Does Competition Among Public Schools Benefit Students and Taxpayers? American Economic Review 90, 1209-1238.

Jepsen, C., Montgomery, M., 2009. Miles to Go Before I Learn: The Effect of Travel Distance on the Mature Person's Choice of a Community College. Journal of Urban Economics 65, $64-73$.

Kemnitz, A., 2007. University Funding Reform, Competition, and Teaching Quality. Journal of Institutional and Theoretical Economics 163, 356-378.

Lazear, E., 2001. Educational Production. Quarterly Journal of Economics 116, 777-803. 
Machin, S., Marie, O., Vujić, S., 2011. The Crime Reducing Effect of Education. Economic Journal 121, 463-484.

Meier, V., 2004. Choosing between School Systems: The Risk of Failure. Finanzarchiv 60, 83-93.

Rauch, J.E., 1993. Productivity gains from geographic concentration of human capital: Evidence from the cities. Journal of Urban Economics 34, 380-400.

Rincke, J., 2006. Competition in the Public School Sector: Evidence on Strategic Interaction among US School Districts. Journal of Urban Economics 59, 352-369.

Rosenthal, S., Strange, W., 2008. The Attenuation of Human Capital Spillovers. Journal of Urban Economics 64, 373-389.

Rothschild, M., White, L., 1995. The Analytics of Pricing Higher Education and Other Services in Which the Customers Are Inputs. Journal of Political Economy 103, 573-586.

Sandström, F., Bergström, F., 2005. School Vouchers in Practice: Competition Will not Hurt You. Journal of Public Economics 89, 351-380.

Shaked, A., Sutton, J., 1982. Relaxing Price Competition Through Product Differentiation. Review of Economic Studies 49, 3-13.

The Economist, 1998, Sep 18th. Making it Pay .

Wößmann, L., 2003. Schooling Resources, Educational Institutions and Student Performance: the International Evidence. Oxford Bulletin of Economics and Statistics 65, 117 170. 